\title{
Dispatching Stochastic Heterogeneous Resources Accounting for Grid and Battery Losses
}

\author{
Eleni Stai, Member, IEEE, Lorenzo Reyes, Member, IEEE, Fabrizio Sossan, Member, IEEE, Jean-Yves Le \\ Boudec, Fellow, IEEE, Mario Paolone, Senior Member, IEEE
}

\begin{abstract}
We compute an optimal day-ahead dispatch plan for distribution networks with stochastic resources and batteries, while accounting for grid and battery losses. We formulate and solve a scenario-based AC Optimal Power Flow (OPF), which is by construction non-convex. We explain why the existing relaxation methods do not apply and we propose a novel iterative scheme, Corrected DistFlow (CoDistFlow), to solve the scenariobased AC OPF problem in radial networks. It uses a modified branch flow model for radial networks with angle relaxation that accounts for line shunt capacitances. At each step, it solves a convex problem based on a modified DistFlow OPF with correction terms for line losses and node voltages. Then, it updates the correction terms using the results of a full load flow. We prove that under a mild condition, a fixed point of CoDistFlow provides an exact solution to the full AC power flow equations. We propose treating battery losses similarly to grid losses by using a single-port electrical equivalent instead of battery efficiencies. We evaluate the performance of the proposed scheme in a simple and real electrical networks. We conclude that grid and battery losses affect the feasibility of the dayahead dispatch plan and show how CoDistFlow can handle them correctly.
\end{abstract}

Index Terms-Dispatch plan; day-ahead; optimal power flow; grid losses; battery models;

$$
\begin{array}{ll}
\text { PCC } & \begin{array}{c}
\text { NOMENCLATURE } \\
\text { Point of Common Coupling. }
\end{array} \\
\text { Indices } & \\
l \in\{1, \cdots, N\} & \text { Index of lines. } \\
i \in\{1, \cdots, N\} & \text { Index of buses/nodes. } \\
\text { up }(l), l & \text { Index of bus at the top and bottom of line } \\
d \in\{1, \cdots, D\} & \text { Index of scenarios. } \\
t \in\{1, \cdots, T\} & \text { Index of time. }
\end{array}
$$

Parameters per line $l$

$\begin{array}{ll}r_{l} & \text { Direct sequence longitudinal resistance. } \\ x_{l} & \text { Direct sequence longitudinal reactance. } \\ b_{l} & \text { Direct sequence shunt susceptance. } \\ \bar{I}_{l} & \text { Ampacity limit (current upper bound). }\end{array}$

Variables per line $l$

$$
\begin{array}{ll}
P_{l}^{d}(t) & \text { Direct sequence active power entering from bus } \\
& \operatorname{up}(l) \text { at time } t \text { and for scenario } d . \\
Q_{l}^{d}(t) & \begin{array}{l}
\text { Direct sequence reactive power entering from bus } \\
\operatorname{up}(l) \text { at time } t \text { and for scenario } d .
\end{array}
\end{array}
$$

The authors are with the École Polytechnique Fédérale de Lausanne (EPFL), Lausanne 1015, Switzerland.

E-mails: \{eleni.stai, lorenzo.reyes, fabrizio.sossan, jean-yves.leboudec, mario.paolone\}@epfl.ch
$S_{l}^{d}(t) \quad$ Direct sequence apparent power entering from bus $\operatorname{up}(l)$ at time $t$ and for scenario $d$.

$f_{l}^{d}(t) \quad$ Square magnitude of the direct sequence current in $z_{l}$ at time $t$ and for scenario $d$.

$i_{l}^{d}(t) \quad$ Current entering the line from the top at time $t$ and for scenario $d$.

$i_{l}^{b, d}(t) \quad$ Current exiting the line at the bottom at time $t$ and for scenario $d$.

Node parameters

$b_{i} \quad$ Sum of all shunt susceptances connected to node $i$.

$\underline{v}, \bar{v} \quad$ Lower and upper node voltage magnitude bounds for the direct sequence.

Variables per node $i$

$v_{i}^{d}(t) \quad$ Square magnitude of the direct sequence voltage, at time $t$ and for scenario $d$.

$p_{i}^{d}(t) \quad$ Active power injection (excluding battery power injections), for scenario $d$ and time $t$.

$q_{i}^{d}(t) \quad$ Reactive power injection (excluding battery power injections), for scenario $d$ and time $t$.

$s_{i}^{d}(t) \quad$ Apparent power injection at bus $i$ (excluding battery power injections), for scenario $d$ and time $t$.

$P^{D P}(t)$ Dispatched active power at the PCC at time $t$.

$Q^{D P}(t)$ Dispatched reactive power at the PCC at time $t$.

$S^{D P}(t)$ Dispatched apparent power at the PCC at time $t$.

Parameters per battery connected at node $i$

$s_{B, i}^{R} \quad$ Rated power.

Variables per battery connected at node $i$

$\operatorname{SoE}_{B, i}^{d}(t) \quad$ State-of-energy (SoE) for time $t$ and scenario $d$.

$\underline{\mathrm{SoE}}_{B, i} \quad$ SoE lower bound.

$\overline{\mathrm{SoE}}_{B, i} \quad$ SoE upper bound.

$p_{B, i}^{+, d}(t) \quad$ Charging power for time $t$ and scenario $d$.

$p_{B, i}^{-, d}(t) \quad$ Discharging power for time $t$ and scenario $d$.

$p_{B, i}^{d}(t) \quad$ Active power for time $t$ and scenario $d$.

$q_{B, i}^{d}(t) \quad$ Reactive power for time $t$ and scenario $d$.

Notation used in Section IV

$\hat{p}_{l}^{d}(t) \quad$ Active power correction term for line $l$, scenario $d$ and time $t$.

$\hat{q}_{l}^{d}(t) \quad$ Reactive power correction term for line $l$, scenario $d$ and time $t$.

$\hat{v}_{l}^{d}(t) \quad$ Voltage square magnitude correction term for line $l$, scenario $d$ and time $t$.

$\tilde{v}_{i}^{d}(t) \quad$ Approximation term for node $i$, scenario $d$ and time $t$. 


\section{General notation}

G $\quad N \times N$ adjacency matrix of the oriented graph of the network excluding the PCC.

|.| Absolute value.

\|. $\quad$ Euclidean norm.

$\Delta t \quad$ Dispatch plan time interval.

$\operatorname{diag}(r) \quad$ Diagonal matrix of vector $r$ whose $l^{\text {th }}$ element is $r_{l}$.

$\|\mathbf{A}\|_{1} \quad$ Induced $l_{1}-$ norm of the matrix $\mathbf{A}$, i.e., $\|\mathbf{A}\|_{1}=$ $\max _{j=1, \ldots, N} \sum_{k=1}^{N}\left|A_{k, j}\right|$.

\section{Collective notation}

$P^{d}(t)$ Column vector of active power for all lines, scenario $d$ and time $t$.

$Q^{d}(t)$ Column vector of reactive power for all lines, scenario $d$ and time $t$.

$v^{d}(t) \quad$ Column vector of voltage square magnitudes for all nodes, scenario $d$ and time $t$.

$p^{d}(t) \quad$ Column vector of node active power injection for all nodes, scenario $d$ and time $t$.

$q^{d}(t) \quad$ Column vector of node reactive power injection for all nodes, scenario $d$ and time $t$.

$s(t, d) \quad$ Nodal injections for all nodes, scenario $d$ and time $t$.

$p_{B}^{d}(t) \quad$ Column vector of battery active power for all buses, scenario $d$ and time $t$.

$q_{B}^{d}(t) \quad$ Column vector of battery reactive power for all buses, scenario $d$ and time $t$.

$s_{B}(t, d)$ Battery powers for all buses, scenario $d$ and time $t$.

$\hat{P}^{d}(t) \quad$ Column vector of active power correction terms for all lines, scenario $d$ and time $t$.

$\hat{Q}^{d}(t) \quad$ Column vector of reactive power correction terms for all lines, scenario $d$ and time $t$.

$\hat{V}^{d}(t)$ Column vector of voltage square magnitude correction terms for all lines, scenario $d$ and time $t$.

$\tilde{V}(t, d)$ Approximation terms for all nodes, scenario $d$ and time $t$.

$E(t, d)$ Electrical state of the grid for all lines/nodes, scenario $d$ and time $t$.

$C(t, d)$ Correction terms for all lines, scenario $d$ and time $t$.

$S \quad$ Apparent power for all lines, scenarios and times.

$v \quad$ Voltage square magnitude for all buses, scenarios and times.

$S^{D P} \quad$ Dispatched apparent power for all times.

$f \quad$ Square magnitude currents flowing through the longitudinal impedances for all lines, scenarios and times.

$S_{B} \quad$ Batteries' charge, discharge and reactive power for all scenarios and times.

$b \quad$ Vector of $b_{i}$ for all buses $i$, excluding the slack bus.

$x \quad$ Vector of reactances for all lines.

\section{INTRODUCTION}

The monotonously increasing deployment of distributed energy resources, if adopted passively, can lead to raising the costs of the investment and operation of power distribution systems, which may ultimately affect the widespread adoption of this technology [1]. Alternatively, distributed resources can be aggregated into a single entity to trade electrical energy or to provide system support services such as dispatchability or reserve [2], [3].

Computing a dispatch plan for a distribution network with stochastic resources and storage devices while accounting for operational constraints and system losses involves an $\mathrm{AC}$ Optimal Power Flow (AC OPF). This problem involves the non-linear power flow equations, thus it is, as well known, nonconvex and hard to solve. There are several proposed methods for the solution of the AC OPF that fall in three categories. First, there are methods that apply relaxation techniques (e.g., [4], [5], [6], [7]) and yield exact solutions (i.e., solutions that coincide with the one of the non-relaxed AC OPF problem), under some stronger conditions [5], [6] or milder conditions [4], [7]. Second, there are methods that apply approximations by modifying the physical description of the power flow equations, e.g., the DC load flow [8], [9] for transmission networks and, most importantly, the DistFlow approximation [10] that has been historically applied to radial distribution networks. In this category, there also exist methods which linearize the AC power flow equations around an operating point, e.g., the load-zero point, as applied in [11], [12]. In addition, [13] adopts an iterative linearization method, where the operating point is updated by the previous iteration. Finally, there are heuristics, e.g., genetic algorithms [14] and nonlinear optimization methods, e.g., Lagrangian-based methods [15], [16] that guarantee only local solutions. In [17], it is shown that under specific conditions the dual gap is zero thus solving the dual problem leads to the global optimal solution.

The relaxation methods [4], [5], [6] do not account for the shunt capacitors of the equivalent two-port $\pi$ line-model, which leads to a solution that might drive the grid to operate into a technically unfeasible point [18]. In [7], the proposed relaxation method takes into account the shunt capacitors, along with considering realistic assumptions with respect to the bounds of the control variables. In this paper, we leverage on the DistFlow approximation that has similar assumptions as [7] but ignores the active/reactive power losses on the longitudinal impedance of the equivalent two-port $\pi$ linemodel.

Several recent papers tackle the AC OPF problem in multiphase unbalanced power grids, e.g., [19], [20], [21], [22], [23]. In [19] and [20], semidefinite programming relaxation is adopted, while [21] proposes a distributed optimization, based on the alternative direction method of multipliers (ADMM) and semidefinite relaxation. The approach of [23] is based on ADMM, for the OPF solution in unbalanced radial grids, where the ADMM subproblems are reduced to either a closed form solution or an eigen-decomposition of a $6 \times 6$ Hermitian matrix. An iterative algorithm is proposed in [22] applying successive convex approximation starting from feasible points, which are determined via another preceding iterative algorithm. Furthemore, the linearization method proposed in [11] can be also applied in multiphase unbalanced power grids.

The uncertainty of the loads and stochastic generation can be handled by means of robust optimization [24], chance- 
constraints [11], [13] or scenario-based optimization [25]. Robust optimization represents uncertainty by intervals and usually minimizes a worst-case value of the objective function, while requiring that the constraints hold for all the values of the uncertain parameters within the considered intervals. Chance-constraints reduce the conservativeness of the robust optimization by requiring that the constraints involving uncertain parameters are satisfied with a specific probability. However, they are more suitable for linear constrained problems [9], [13], [25], otherwise they become hard to approximate and usually samples (i.e., scenarios) are used for this approximation e.g., [11], [25], [26]. The scenario-based approach uses realization scenarios of the uncertain parameters derived e.g., from past measurements in combination with predictions [3], [27], requiring the satisfaction of the constraints for every scenario. It renders the uncertainty easier to handle in the problem formulation, and allows for general convex constraints. Note that for an appropriate choice of the number of the scenarios, the solution of the scenario-based problem is a feasible solution of a corresponding chance-constrained one [25] (with a confidence level). These methods have been applied to the unit commitment problem [28], [24] under uncertain resources and to the OPF problem, in several cases with renewable generation and storage, [3], [27], [29], [11], [13]. Based on the above discussion, we apply scenario-based optimization, which, according to [30], models appropriately the uncertainty of stochastic resources while representing any existing time correlation structure.

We propose a generic iterative scheme, denoted as Corrected DistFlow (CoDistFlow), for solving a scenario-based AC OPF in radial networks with stochastic resources and controllable batteries, aiming to compute a day-ahead dispatch plan at the PCC, by accounting for both grid and battery losses. The scheme is based on the branch-flow model for radial networks with angle relaxation, which is able to account for the presence of line shunt capacitances [7]. It consists of two modules executed sequentially at each iteration until convergence. The first module is called Improved DistFlow (iDF). It solves a convex problem using the DistFlow approximation but with appropriate correction and approximation terms for losses and voltages, mimicking the optimization terms in the non-linear power flow equations ignored by DistFlow. The second module solves appropriate load flow (LF) problems for computing the correction and approximation terms with inputs the battery trajectories obtained via $\mathrm{iDF}$ in the previous iteration. We prove that, under mild assumptions, a fixed point of CoDistFlow (i.e., after convergence in the limit) is a solution of the exact power flow equations and satisfies the exact operational constraints. The numerical evaluations illustrate that convergence takes place in few iterations. Regarding the realistic behavior of batteries, we propose to treat their losses as those taking place in the longitudinal resistance of a line. To do that, we use the AC single-port circuit equivalent of the battery. Then, CoDistFlow treats the battery losses similarly to the non-linearities of the power flow equations. Importantly this modeling of the battery allows for an exact relaxation of the non-convex battery constraint applied to avoid its simultaneous charging and discharging. We evaluate the proposed scheme in a 4-bus network and in a real grid. As main outcome, we show that not considering the grid and battery losses could lead to a failure of the dispatch plan in realistic scenarios. Furthermore we perform comparative evaluations with an iterative algorithm that linearizes the $\mathrm{AC}$ power flow equations around an operating point, illustrating the advantages of CoDistFlow. Importantly, CoDistFlow is a method of independent interest for solving any AC OPF problem (e.g., even non scenario-based or without battery storage).

In general, compared with similar works in literature:

- CoDistFlow computes a day-ahead dispatch plan at the PCC by appropriately accounting for both grid and battery losses. It is shown that non-considering the grid and battery losses may significantly impact the mismatch between the dispatch plan and the actual realization at the PCC for a realization of the uncertain prosumption. CoDistFlow demonstrates to properly handle this aspect. To the best of our knowledge this is the first work in literature showing such results.

- Compared with other linearization algorithms in literature, CoDistFlow linearizes the power flow equations based on DistFlow and then it iteratively corrects the linearization using feedback from the previous iteration on the line losses and node voltages that can be computed very efficiently via load flow. Most of the linearization methods in literature linearize the power flow equations around an operating point using the Jacobian matrix. Then, they correct the linearization by updating the operating point using the optimal solution of the previous iteration, which is not always efficient as it is shown in our results. The linearization methods of [11], [12] are performed only once around the load-zero operating point. Furthermore, the iterative linearization of [13] is applied only in order to simplify the chance-constraints for the voltage and the current and not to solve the AC OPF itself. An advantage of CoDistFlow is that it converges in only few iterations (shown via simulations), while at a fixed point, the derived electrical state of the grid is proven to satisfy the exact AC power flow equations and the exact operational constraints.

- Thirdly, CoDistFlow applies scenario-based optimization which renders the uncertainty easy to handle in the problem formulation and, more importantly, generic in its distribution (e.g., non-Gaussian), while it allows for general convex constraints. By requiring the satisfaction of all the constraints by all the scenarios, if an appropriate number of scenarios is considered, it provides a feasible solution to a corresponding chance-constrained problem that applies probabilistic (chance) constraints for all constraints types, e.g., voltage magnitude, current magnitude, battery state-of-energy, power factor, contrary to related papers in literature applying chance-constraints only for specific types of constraints e.g., [11], [13], [9]. Also, we show that the existing relaxation methods for solving the AC OPF yielding exact solutions, e.g., [4], [7], do not apply in case of scenario-based optimization 


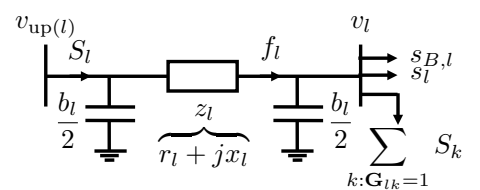

Fig. 1: Notation for the $\pi$ model of a line.

and CoDistFlow is proposed as a solution to this issue.

- Finally, CoDistFlow can efficiently handle a more realistic battery model without impact on its computational complexity, as explained above.

The paper is organized as follows. Section II introduces the assumptions on the system model, the battery models and formulates the problem under consideration. Section III explains why current relaxation techniques yielding exact solutions are not compatible with scenario-based optimization. Section IV presents and analyzes CoDistFlow, and Section $\mathrm{V}$ presents the evaluation and comparison results. Finally, Section VI discusses possible extensions of CoDistFlow and Section VII concludes the paper.

\section{System Model And Problem Formulation}

\section{A. Assumptions}

We consider a balanced and transposed radial distribution network where the PCC, between the local distribution network and the upstream power grid, is at index 0 and it is assumed as the slack bus with $v_{0}^{d}(t)=1 \mathrm{pu}, \forall t, d$. Distribution lines are represented by their single-phase direct sequence equivalent $\pi$ models (Fig. 1). The node at the top of line $l$ (i.e., electrically closer to the PCC), is denoted as $\operatorname{up}(l)$, and the node at the bottom as $l$. The adjacency matrix $\mathbf{G}$ is characterized by $\mathbf{G}_{k, l}=1$ for two buses $k, l \neq 0$, if $k=\operatorname{up}(l)$, otherwise $\mathbf{G}_{k, l}=0$. Also let us consider that, for every battery system, $p_{B, i}^{+, d}(t), p_{B, i}^{-, d}(t) \geq 0$, with $p_{B, i}^{d}(t)=p_{B, i}^{+, d}(t)-p_{B, i}^{-, d}(t)$ and $\left(p_{B, i}^{+, d}(t)\right)^{2}+\left(p_{B, i}^{-, d}(t)\right)^{2}+\left(q_{B, i}^{d}(t)\right)^{2} \leq\left(s_{B, i}^{R}\right)^{2}$.

In the following, we formulate the scenario-based AC OPF for a radial distribution network with intermittent renewable energy sources and battery storage. This formulation refers to a general radial distribution network where PCC lies at the top of the line $l=1$.

\section{B. Power Flow Equations \& Constraints}

Here, we provide the power flow equations for the branchflow model using the so-called "angle relaxation" [6] with transverse elements [7]. The DistFlow approximation is based on the angle-relaxed $\mathrm{AC} \mathrm{OPF} \mathrm{[10],} \mathrm{in} \mathrm{which,} \mathrm{the} \mathrm{power} \mathrm{flow}$ equations, along with the corresponding voltage and current constraints, are expressed without using the voltage (and current) angles. Note that, in case of radial (tree) networks, the angle relaxation [6] is just a bijective change of variables. Thus, the angles can be recovered as shown in [6] and the solution of the angle-relaxed problem is also a solution of the non-angle-relaxed AC OPF. Next, we provide the constraints of the considered AC OPF divided in specific categories. a) Power Flow Equations defined $\forall t, d, l$ :

$$
\begin{array}{r}
P_{l}^{d}(t)=\sum_{k: \mathbf{G}_{l k}=1} P_{k}^{d}(t)+p_{l}^{d}(t)+p_{B, l}^{d}(t)+r_{l} f_{l}^{d}(t), \\
Q_{l}^{d}(t)=\sum_{k: \mathbf{G}_{l k}=1} Q_{k}^{d}(t)+q_{l}^{d}(t)+q_{B, l}^{d}(t) \\
-\left(v_{\operatorname{up}(l)}^{d}(t)+v_{l}^{d}(t)\right) b_{l} / 2+x_{l} f_{l}^{d}(t),
\end{array}
$$

$$
f_{l}^{d}(t)=\left\|S_{l}^{d}(t)+j \frac{v_{\mathrm{up}(l)}^{d}(t) b_{l}}{2}\right\|^{2} / v_{\mathrm{up}(l)}^{d}(t) .
$$

b) Voltage Constraints, defined $\forall t, d, l, i$ :

$$
\begin{aligned}
v_{l}^{d}(t)=v_{\mathrm{up}(l)}^{d}(t)-2 \Re\left\{z_{l}^{*}\left(S_{l}^{d}(t)+j v_{\mathrm{up}(l)}^{d}(t) \frac{b_{l}}{2}\right)\right\} & +\left\|z_{l}\right\|^{2} f_{l}^{d}(t), \\
v_{\mathrm{up}(1)}^{d}(t)=1, \underline{v}^{2} \leq v_{i}^{d}(t) \leq & \bar{v}^{2} .
\end{aligned}
$$

c) Power Factor Constraints at the PCC:

$$
\left|P_{1}^{d}(t)\right| /\left\|S_{1}^{d}(t)\right\| \geq \cos (\phi)_{\min }, \forall t, d,
$$

where $\cos (\phi)_{\min }$ stands for the minimum allowed power factor.

d) Ampacity Constraints, defined $\forall t, d, l$ :

$$
\begin{array}{r}
\Re\left\{i_{l}^{d}(t)\right\}=\frac{P_{l}^{d}(t)}{\sqrt{v_{\mathrm{up}(l)}^{d}(t)}}, \quad \Im\left\{i_{l}^{d}(t)\right\}=\frac{Q_{l}^{d}(t)}{\sqrt{v_{\mathrm{up}(l)}^{d}(t)}}, \\
\Re\left\{i_{l}^{b, d}(t)\right\}=\left(P_{l}^{d}(t)-r_{l} f_{l}^{d}(t)\right) / \sqrt{v_{l}^{d}(t)}, \\
\Im\left\{i_{l}^{b, d}(t)\right\}=\Im\left\{i_{l}^{d}(t)\right\} \\
+\left(v_{\mathrm{up}(l)}^{d}(t)+v_{l}^{d}(t)\right) \frac{b_{l}}{2 \sqrt{v_{l}^{d}(t)}}-\frac{x_{l} f_{l}^{d}(t)}{\sqrt{v_{l}^{d}(t)}}, \\
\left\|i_{l}^{d}(t)\right\| \leq \bar{I}_{l},\left\|i_{l}^{b, d}(t)\right\| \leq \bar{I}_{l} .
\end{array}
$$

e) Battery Constraints, defined $\forall t, d, i$ :

$$
\begin{array}{r}
\operatorname{SoE}_{B, i}^{d}(t+1)=\operatorname{SoE}_{B, i}^{d}(t)+\left(\eta_{1} p_{B, i}^{+, d}(t)-\eta_{2} p_{B, i}^{-, d}(t)\right) \Delta t \\
a_{B} \underline{\operatorname{SoE}}_{B, i} \leq \operatorname{SoE}_{B, i}^{d}(t) \leq\left(1-a_{B}\right) \overline{\operatorname{SoE}}_{B, i} \\
\left(p_{B, i}^{+, d}(t)\right)^{2}+\left(p_{B, i}^{-, d}(t)\right)^{2}+\left(q_{B, i}^{d}(t)\right)^{2} \leq\left(s_{B, i}^{R}\right)^{2} \\
p_{B, i}^{+, d}(t) p_{B, i}^{-, d}(t)=0
\end{array}
$$

where $\eta_{1}, \eta_{2}$ will be determined based on the battery model under consideration (see Section II-E) and $0 \leq a_{B} \leq 1$ is a constant parameter used to define a margin on the SoE limits. The constraint of Eq. (14) serves the purpose of avoiding the simultaneous charge and discharge of the battery.

The formulated problem is non-convex due to the constraints of Eqs. (3), (7)-(10), (6) and (14). In the following, (6) will be replaced by a convex soft constraint that provides more flexibility in terms of finding feasible solutions. Also, (14) can be accounted by appropriately augmenting the objective function (this aspect is discussed in Section II-F). The first two constraints' sets are therefore the main reasons for the non-convexity of this problem formulation. 


\section{Initial Objective Function}

The objective function is defined such that the optimal solution trades-off the following five objectives. First, there is a penalty on the state-of-energy of the battery, $\phi\left(\operatorname{SoE}_{B, i}^{d}(t)\right)$; it expresses the preference that $\operatorname{SoE}_{B, i}^{d}(t) \in\left[\underline{\mathrm{E}}_{B, i}, \overline{\mathrm{E}}_{B, i}\right]$, with $\underline{\mathrm{E}}_{B, i} \geq \underline{\mathrm{SoE}}_{B, i}$ and $\overline{\mathrm{SoE}}_{B, i} \geq \overline{\mathrm{E}}_{B, i}$. This serves the purpose of reducing the battery deep of discharge and limiting the battery degradation, along with introducing more flexibility for handling uncertainties in the real-time operation that are not accounted for in day-ahead via the scenarios. Specifically,

$\phi\left(\operatorname{SoE}_{B, i}^{d}(t)\right)=\max \left(\underline{\mathrm{E}}_{B, i}-\operatorname{SoE}_{B, i}^{d}(t), 0, \operatorname{SoE}_{B, i}^{d}(t)-\overline{\mathrm{E}}_{B, i}\right)$

The second objective minimizes the reactive power at the PCC $\left(\left|Q_{1}^{d}(t)\right|\right)$ that serves the purpose of maximizing the power factor at the PCC for every scenario [31]. The third objective minimizes the active power exchanged with the upstream power grid. The fourth goal is to maximize the power export to the main grid. The fifth objective relates to the error between the obtained dispatch plan and the optimal power at the PCC for every scenario. This is a crucial objective for determining a dispatch plan such that all the considered scenarios of the uncertain parameters will be able to follow in real-time by using the batteries. The individual objectives are weighted by the positive constants $w_{i}, i=1, \ldots, 5^{1}$. Finally, the objective function is a weighted average over all scenarios, where the weight $\lambda_{d}$ is the probability of occurrence of scenario $d$, with $\sum_{d} \lambda_{d}=1$. Thus, the initial version of the objective function to be minimized is:

$$
\begin{aligned}
w_{1} \sum_{d, t, i} \lambda_{d} \phi & \left(\operatorname{SoE}_{B, i}^{d}(t)\right)+w_{2} \sum_{d, t} \lambda_{d}\left|Q_{1}^{d}(t)\right|+w_{3} \sum_{d, t} \lambda_{d}\left|P_{1}^{d}(t)\right| \\
& +w_{4} \sum_{d, t} \lambda_{d} P_{1}^{d}(t)+w_{5} \sum_{d, t} \lambda_{d}\left\|S_{1}^{d}(t)-S^{D P}(t)\right\|^{2} .
\end{aligned}
$$

In order to remove the non-linearities of the penalty function $\phi($.$) leading to an equivalent problem formulation, we$ introduce the slack optimization variables $E_{B, i}^{d}(t)$ and (i) we replace the term $w_{1} \sum_{d, t, i} \lambda_{d} \phi\left(\operatorname{SoE}_{B, i}^{d}(t)\right)$ in the objective function (Eq. (16)) with $w_{1} \sum_{d, t, i} \lambda_{d} E_{B, i}^{d}(t)$, (ii) we add the following battery constraints, $\forall i, d, t$,:

$$
\begin{aligned}
\underline{\mathrm{E}}_{B, i}-\operatorname{SoE}_{B, i}^{d}(t) & \leq E_{B, i}^{d}(t), \\
0 & \leq E_{B, i}^{d}(t), \\
-\overline{\mathrm{E}}_{B, i}+\operatorname{SoE}_{B, i}^{d}(t) & \leq E_{B, i}^{d}(t) .
\end{aligned}
$$

Note that, in a similar way, we can remove the non-linearities of the objectives weighted by $w_{2}$ and $w_{3}$, which is not presented here for the ease of presentation.

In the following, we further enhance the objective function for the purpose of relaxing non-convex constraints.

\footnotetext{
${ }^{1}$ The weight values are assigned based on the importance of each individual objective. Specifically, we should assign $w_{5}>0$ and high enough so that the derived optimal dispatch plan will be followed in the real time operation (e.g., if participating at the market with our feeder as the dispatchable resource). Also, based on the discussion above, we should assign $w_{1}>0$ and $w_{2}>0$. Finally, $w_{4}-w_{3}$ can be interpreted as the price received when exporting power to the upstream power grid and $w_{3}+w_{4}$ as the price paid when importing power to the local distribution grid by the upstream grid.
}

\section{Soft Power Factor Constraint}

In this section, we replace the hard power factor constraint of Eq. (6) with a soft and convex constraint form. The latter is essential as the network acts as a prosumer, thus the dispatched active power at the PCC could take zero values for some time intervals, leading to infeasibility under a hard constraint.

Let us introduce the optimization variables $P^{+, d}(t), P^{-, d}(t), \forall t, d$. In the objective function (Eq. (16)) we add the term $w_{6} \sum_{d, t} \lambda_{d}\left(\left(P^{+, d}(t)\right)^{2}+\left(P^{-, d}(t)\right)^{2}\right)$, where $w_{6} \geq 0$ weights the importance of satisfying the power factor constraints. Also, instead of the constraint of Eq. (6), we add the following set of constraints, $\forall t, d$ :

$$
\begin{array}{r}
P^{+, d}(t)+P^{-, d}(t) \geq Q_{1}^{d}(t) \tan \left(\pi / 2-\phi_{m}\right), \\
P^{+, d}(t)+P^{-, d}(t) \geq-Q_{1}^{d}(t) \tan \left(\pi / 2-\phi_{m}\right), \\
P_{1}^{d}(t)=P^{+, d}(t)-P^{-, d}(t), P^{+, d}(t) \geq 0, P^{-, d}(t) \geq 0,
\end{array}
$$

where $\phi_{m}$ is the phase-shift corresponding to $\cos (\phi)_{\min }$. The solution subject to the above set of constraints might not satisfy Eq. (6), when the problem is not feasible subject to Eq. (6) or when the rest objectives are more important for minimization purposes (e.g., when $w_{6}$ is small). The former may be the case when the active power at the PCC takes values close to zero, i.e., in presence of distributed renewable energy sources. In this case we may not be able to obtain an optimal dispatch plan under Eq. (6), while on the contrary there is a solution if adopting the above relaxation. Under feasibility, the satisfaction of Eq. (6) can be enforced by assigning higher values to the weight value $w_{6}$, compared to the rest of the weight values. In addition, the second summand of Eq. (16) aims at maximizing the power factor at the PCC (by minimizing the absolute value of the reactive power at the PCC) which further enforces the satisfaction of Eq. (6) in case of feasibility. The following result is shown:

Proposition 1: Assume that $w_{i}=0, \forall i \neq 6$, the optimal solution of the problem of minimizing (16) subject to (1)(5), (7)-(14), (20)-(22) (and also the optimal solution of the problem defined in Section II-G), satisfies Eq. (6), if this problem is feasible subject to Eq. (6).

Proof: The satisfaction of the soft constraints (Eqs. (20)(22)) with either $P^{+, d}(t)>0$ or $P^{-, d}(t)>0$ is equivalent to the satisfaction of Eq. (6). To make this clearer, from Fig. 2, we observe that Eq. (6) is equivalent to

$$
\frac{\left|P_{1}^{d}(t)\right|}{\left|Q_{1}^{d}(t)\right|} \geq \tan \left(\pi / 2-\phi_{m}\right),
$$

i.e., to

$$
\begin{gathered}
\left|P_{1}^{d}(t)\right| \geq Q_{1}^{d}(t) \tan \left(\pi / 2-\phi_{m}\right) \text { and } \\
\left|P_{1}^{d}(t)\right| \geq-Q_{1}^{d}(t) \tan \left(\pi / 2-\phi_{m}\right) .
\end{gathered}
$$

We write $P_{1}^{d}(t)=P^{+, d}(t)-P^{-, d}(t)$, with $P^{+, d}(t) \geq$ $0, P^{-, d}(t) \geq 0$. Then, if either $P^{+, d}(t)>0$ or $P^{-, d}(t)>0$, the previous two inequalities become the Eqs. (20)-(21). This is also the choice of $P^{+, d}(t), P^{-, d}(t)$ (either $P^{+, d}(t)>0$ or $P^{-, d}(t)>0$ ) that minimizes the objective function with $w_{i}=0, \forall i \neq 6$ (optimal solution), since $P^{+, d}(t), P^{-, d}(t)$ appear only in constraints (20)-(22). This proves the proposition. 


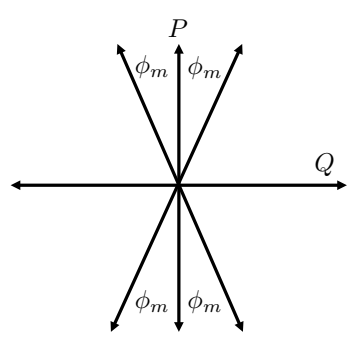

Fig. 2: Illustration used for the derivation of the soft power factor constraint.

\section{E. Modeling of Lossy Batteries}

In order to account for battery charge and discharge losses, an efficiency-based model with constant efficiencies is conventionally used [3]. This model is very accurate for constant power-exchanges, but less accurate when the exchanged power is variable. As an alternative, a resistance-based model can be used and can be easily included in our problem formulation. Note that, we do not model the self-discharge of the battery as it is negligible for the time-scale of interest [3]. For simplicity, let us denote the active power injected into the grid as $p_{B}^{+}$and $p_{B}^{-}$(for charging and discharging respectively), whereas on the battery side, $p_{d c}^{+}$and $p_{d c}^{-}$, with same interpretation. Both models are presented next.

1) Conventional Efficiency Model: The conventional model states that $p_{d c}^{+}=\eta_{1} p_{B}^{+} \quad\left(\eta_{1}<1\right), p_{d c}^{-}=\eta_{2} p_{B}^{-} \quad\left(\eta_{2}>1\right)$, where $\eta_{1}$ and $\eta_{2}$ are the directional charging and discharging efficiencies respectively. Several approaches can be used in order to evaluate $\eta_{1}$ and $\eta_{2}$. The common practice is to assess them experimentally, by computing an average efficiency in a wide range of operational conditions. It is also typical to consider that battery losses are symmetric, namely, $\eta_{1} \eta_{2}=1$.

2) Resistance Model: First, we assume that all losses in the battery can be represented by a single resistance. Furthermore, we assume that the internal losses of the battery pack are more important than those of the power converter. Then, the battery can be represented by an ideal storage $\left(\eta_{1}=1, \eta_{2}=1\right)$ with injection $p_{d c}$ in a virtual node that is connected to a purely resistive line (no shunt elements). As the reactive power is entirely product of the power conversion, the equivalent injection, $Q$, is connected at the real node as shown in Fig. $3^{2}$.

This simplification enables us to directly integrate this equivalent model into the admittance matrix of the system (increasing its dimension by the corresponding number of batteries in the grid), thus without affecting the problem computational complexity. Specifically, the non-linearity introduced by the additional resistive line in the AC OPF will be treated similarly to the non-linearities of the power flow equations at every other line (Section IV). Note that, we impose neither ampacity constraints to this line, nor voltage constraints at the virtual node.

As with the efficiency model, the value of $R_{B}$ can be assessed experimentally. Here, we assess both models' parameters from an accurate TTC-model presented in [3].

\footnotetext{
${ }^{2}$ Note that a more generic model would simply include an additional resistance that models the power converter losses.
}

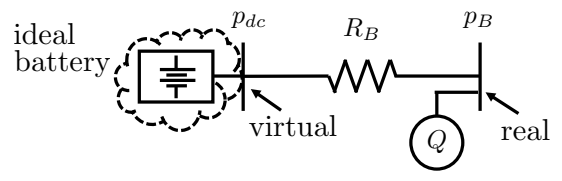

Fig. 3: Battery equivalent resistance model.

\section{F. Relaxation of the Constraint of Eq. (14)}

In this section, we propose the enhancement of the objective function (Eq. (16)) by an extra linear term, in order to alleviate the non-convex constraint of Eq. (14). Specifically, we add the term $w_{7} \sum_{d, t, i} \lambda_{d}\left(p_{B, i}^{+, d}(t)+p_{B, i}^{-, d}(t)\right)$ in Eq. (16), with $w_{7}>0$, and we remove the constraint of Eq. (14). After all the proposed relaxations and equivalent modifications, the objective function is written as:

$$
\begin{array}{r}
w_{1} \sum_{d, t, i} \lambda_{d} E_{B, i}^{d}(t)+w_{2} \sum_{d, t} \lambda_{d}\left|Q_{1}^{d}(t)\right|+w_{3} \sum_{d, t} \lambda_{d}\left|P_{1}^{d}(t)\right| \\
+w_{4} \sum_{d, t} \lambda_{d} P_{1}^{d}(t)+w_{5} \sum_{d, t} \lambda_{d}\left\|S_{1}^{d}(t)-S^{D P}(t)\right\|^{2} \\
+w_{6} \sum_{d, t} \lambda_{d}\left(\left(P^{+, d}(t)\right)^{2}+\left(P^{-, d}(t)\right)^{2}\right) \\
+w_{7} \sum_{d, t, i} \lambda_{d}\left(p_{B, i}^{+, d}(t)+p_{B, i}^{-, d}(t)\right) .
\end{array}
$$

Theorem 1: If $w_{7}>0$ and the resistance model is applied for the battery, then in the optimal solution of the problem defined in Section II-G the battery charge and discharge power values $\left(p_{B, i}^{+, d}(t), p_{B, i}^{-, d}(t), \forall i, d, t\right)$ satisfy the constraint of Eq. (14).

Proof: The proof is by contradiction. Consider an optimal solution, and assume that $p_{B, i}^{+, d}(t)>0, p_{B, i}^{-, d}(t)>0$, for some time $t$, scenario $d$ and node $i$. Also, assume for the moment that $p_{B, i}^{+, d}(t)>p_{B, i}^{-, d}(t)$. Then, according to the battery resistance model, the power injected into the virtual line connecting the battery to the grid is $p_{B, i}^{d}(t)=p_{B, i}^{+, d}(t)-p_{B, i}^{-, d}(t)$ and the change in the corresponding battery's state-of-energy is equal to $\Delta \operatorname{SoE}_{B, i}^{d}(t)=p_{B, i}^{d}(t) \Delta t$.

We now build another feasible solution which is identical to the optimal solution except for $\tilde{p}_{B, i}^{+, d}(t)=p_{B, i}^{+, d}(t)-p_{B, i}^{-, d}(t)>$ 0 and $\tilde{p}_{B, i}^{-, d}(t)=0$. This solution leads to the same power injected into the virtual line connecting the battery to the grid and the same battery state-of-energy as the optimal one, therefore it is feasible. Note that it also satisfies the constraint of Eq. (13) since the latter is satisfied by the optimal solution.

Furthermore, the difference in cost incurred by considering $p_{B, i}^{+, d}(t), p_{B, i}^{-, d}(t)$ instead of $\tilde{p}_{B, i}^{+, d}(t), \tilde{p}_{B, i}^{-, d}(t)$ is $w_{7} \lambda_{d}\left(p_{B, i}^{+, d}(t)+\right.$ $\left.p_{B, i}^{-, d}(t)-\tilde{p}_{B, i}^{+, d}(t)-\tilde{p}_{B, i}^{-, d}(t)\right)=2 w_{7} \lambda_{d} p_{B, i}^{-, d}(t)>0$. Thus, this new feasible solution has a lower value of the objective, which contradicts the optimality of the original solution. The case $0<p_{B, i}^{+, d}(t)<p_{B, i}^{-, d}(t)$ is handled similarly with $\tilde{p}_{B, i}^{+, d}(t)=0$ and $\tilde{p}_{B, i}^{-, d}(t)=p_{B, i}^{-, d}(t)-p_{B, i}^{+, d}(t)>0$.

Remark 1: The above theorem is not true when the conventional efficiency model is applied for the battery. Specifically, in this case, it may happen that both $p_{B, i}^{+, d}(t)>0$ and $p_{B, i}^{-, d}(t)>0$ in the optimal solution, when the battery stateof-energy is close to its hard upper bound, $\left(1-a_{B}\right) \overline{\operatorname{SoE}}_{B, i}$. 
Using the same battery charge and discharge power values as in the proof of Theorem $1, p_{B, i}^{+, d}(t), p_{B, i}^{-, d}(t)$ will lead to a change in the corresponding battery's state-of-energy equal to $\Delta \operatorname{SoE}_{B, i}^{d}(t)=\left(\eta_{1} p_{B, i}^{+, d}(t)-\eta_{2} p_{B, i}^{-, d}(t)\right) \Delta t$, while $\tilde{p}_{B, i}^{+, d}(t), \tilde{p}_{B, i}^{-, d}(t)$ will lead to a change in the corresponding battery's state-of-energy equal to $\Delta \tilde{\operatorname{SoE}}_{B, i}^{d}(t)=\eta_{1}\left(p_{B, i}^{+, d}(t)-\right.$ $\left.p_{B, i}^{-, d}(t)\right) \Delta t>\Delta \operatorname{SoE}_{B, i}^{d}(t)$. Therefore, only in the case that the battery's state-of-energy is close to its hard upper bound, the latter battery power values may render the solution infeasible in terms of the battery constraints, and the former battery power values may be chosen at the optimal solution.

However, in case of the conventional efficiency battery model we relax the non-convex constraint of Eq. (14) in the same way as for the resistance model, since due to the penalty function, $\phi\left(\operatorname{SoE}_{B, i}^{d}(t)\right)$, weighted by $w_{1}$ in the objective function, it becomes less probable that at the optimal solution the battery's state-of-energy will be close to its hard upper bound. In the latter case, $\phi\left(\operatorname{SoE}_{B, i}^{d}(t)\right)$ becomes non-zero, increasing linearly with the distance of $\operatorname{SoE}_{B, i}^{d}(t)$ from its preferable upper bound, $\overline{\mathrm{E}}_{B, i}$. For the conventional efficiency battery model, it is also assumed that $w_{7}>w_{1}$. If the latter is not true, $\tilde{p}_{B, i}^{+, d}(t), \tilde{p}_{B, i}^{-, d}(t)$ may induce a higher cost than $p_{B, i}^{+, d}(t), p_{B, i}^{-, d}(t)$ when the battery state-of-energy is close to its preferable upper bound $\overline{\mathrm{E}}_{B, i}$, and thus the latter will be chosen at the optimal solution.

Note that the above issue does not happen when the battery state-of-energy is close to its hard lower bound, $a_{B} \underline{\operatorname{SoE}}_{B, i}$. Indeed, let us assume that at the optimal solution, $p_{B, i}^{+, d}(t)>0, p_{B, i}^{-, d}(t)>0$ for some time $t$ at node $i$ and scenario $d$. Also, assume that $p_{B, i}^{+, d}(t)<p_{B, i}^{-, d}(t)$. Then, $\Delta \operatorname{SoE}_{B, i}^{d}(t)=\left(\eta_{1} p_{B, i}^{+, d}(t)-\eta_{2} p_{B, i}^{-, d}(t)\right) \Delta t$. We define $\hat{p}_{B, i}^{-, d}(t)=-p_{B, i}^{+, d}(t)+p_{B, i}^{-, d}(t)>0$ and $\hat{p}_{B, i}^{+, d}(t)=0$. Then, $\Delta \hat{\operatorname{SoE}}_{B, i}^{d}(t)=\eta_{2}\left(p_{B, i}^{+, d}(t)-p_{B, i}^{-, d}(t)\right) \Delta t>\Delta \operatorname{SoE}_{B, i}^{d}(t)$. Thus, if a solution is feasible for $p_{B, i}^{+, d}(t), p_{B, i}^{-, d}(t)$, it will be also feasible for $\hat{p}_{B, i}^{+, d}(t), \hat{p}_{B, i}^{-, d}(t)$ which reduces less the battery's state-of-energy and induces a lower cost in the objective function, contradicting the fact that $p_{B, i}^{+, d}(t)>0, p_{B, i}^{-, d}(t)>0$ is optimal at time $t$ for scenario $d$ and bus $i$.

Remark 2: The analysis in this section indicates an important aspect of choosing the resistance model for the battery, since it allows the exact relaxation of the non-convex constraint of Eq. (14), while it is also handled very efficiently by our proposed algorithmic solution of the scenario-based AC OPF in Section IV as explained in Section II-E2.

Remark 3: The penalty term $w_{7} \sum_{d, t, i} \lambda_{d}\left(p_{B, i}^{+, d}(t)+p_{B, i}^{-, d}(t)\right)$ can be used to serve two goals: (i) it makes the relaxation of Eq. (14) exact with the battery resistance model and (ii) it penalizes the number of battery charge or discharge cycles [32]. As a result, if applying the resistance model for the battery, $w_{7}$ can be assigned a very small positive value if one does not aim to penalize battery cycling or a larger positive value in the opposite case.

\section{G. AC OPF Problem Formulation}

The non-convex AC OPF is formulated as follows:

$$
\begin{array}{r}
\min _{S, S_{B}, v, S^{D P}, f}(25), \\
\text { s.t. } \forall t, d, l, \\
(1)-(5),(7)-(13),(17)-(22)
\end{array}
$$

We handle two versions of problem (27), one per each battery model (Section II-E). For the efficiency battery model, we assign $\eta_{1}<1, \eta_{2}>1$, while for the resistance model we assign $\eta_{1}=\eta_{2}=1$ and one additional line per battery in the grid, by using the equivalent model of Fig. 3 .

\section{H. Determination of the Number of Scenarios}

In this section, we study the number of scenarios, $D$, that should be included in the optimization problem of Eqs. (26)(27) aiming to achieve a target upper bound for the probability of constraint violation by the optimal solution at a random realization of the uncertain parameters. For this purpose, we will introduce an abstract formulation of our optimization problem. Let $u \in \mathbb{R}^{2 T}$ denote the collection of global control variables (i.e., the dispatch plan), $x$ the collection of control variables that depend on the scenario (i.e., $S, S_{B}, v, f$ ), and $w \in \mathcal{W} \subset \mathbb{R}^{2 T N}$ the uncertain parameters (i.e., the nodal injections for all times). We define an indicator function as $H(u, w)=1$ if there does not exist an $x$ such that all the constraints of Eq. (27) are satisfied for $u$ and a realization of $w$ and $H(u, w)=0$ if there exists an $x$ such that the constraints are satisfied. Also, let $w_{1: D}=\left\{w_{i}: i \in\{1: D\}\right\}$ be the set of scenarios (i.e., realizations of the nodal injections), and define $\mathcal{U}_{w_{1: D}}=\left\{u: H\left(u, w_{i}\right)=0, \forall i \in\{1: D\}\right\}$. Let also $\bar{f}(u)=\mathbb{E}[f(u, x)]$ be the objective function of Eq. (26) where $\mathbb{E}[$.$] denotes the expected value. Then, since we will apply the$ analysis of [25], following its requirements for linear objective function and convex constraints, the optimization problem of Eqs. (26)-(27) is expressed as:

$$
\min _{u, y} y, \text { s.t. } \bar{f}(u) \leq y, u \in \mathcal{U}_{w_{1: D}} .
$$

Then, we define the set $\mathcal{U}(\epsilon)=\{u: \mathbb{P}(w \in \mathcal{W}, H(u, w)=$ $1) \leq \epsilon\}$, where $\epsilon \in(0,1)$, a desirable probability of (a single) constraint violation. We want to determine the number of scenarios, $D$, such that the solution $\left(u^{*}, y^{*}\right)$ of the problem (28) is also a solution of the problem (29) that follows, with a confidence level $1-\alpha \geq 0, \alpha \in(0,1)$ :

$$
\min _{u, y} y, \text { s.t. } \bar{f}(u) \leq y, u \in \mathcal{U}(\epsilon) .
$$

According to [25], given $\alpha, \epsilon$, if

$$
D \geq \frac{2}{\epsilon} \ln \frac{1}{\alpha}+2 n+\frac{2 n}{\epsilon} \ln \frac{2}{\epsilon},
$$

where $n$ is the number of control variables $u$ plus one (for the extra optimization variable $y$ ), i.e., $2 T+1$, then,

$$
\mathbb{P}^{D}\left(\mathbb{P}\left(w \in \mathcal{W}, H\left(u^{*}, w\right)=1\right) \leq \epsilon\right) \geq 1-\alpha,
$$

where $\mathbb{P}^{D}=\mathbb{P} \times \mathbb{P} \ldots \times \mathbb{P}, D$ times, is the probability of selecting a bad set of $D$ scenarios that will not lead to a $u^{*}, y^{*}$ being a solution of the Problem (29). Note that Eq. (30) is 
probability independent and applies also in the case that the scenarios derive from observations.

Following the above, in this work based on desired values of $\alpha$ and $\epsilon$, we use Eq. (30) to determine the number of scenarios, $D$, that should be considered in problem (28). Since for low values of $\epsilon, D$ attains a large value, in order to handle the emerging computational complexity issues, we have performed scenario reduction. Our scenario reduction method is based on the concept of probability distance and follows the lines of the methodology in [33], using the Chebyshev distance to determine the similarity of two scenarios. According to this methodology, the reduced set of scenarios lies close in probability distance to the initial set including all scenarios. Such a scenario reduction methodology is applied also in [34] for stochastic variables of electrical systems. In Section V, we provide details on the numerical values assigned to $\alpha, \epsilon, D$ and the number of scenarios applied after reduction.

Since the bound on the probability of violation obtained above tends to be loose, and since we perform scenario reduction, we compute a refined estimate of the probability $\mathbb{P}\left(w \in \mathcal{W}, H\left(u^{*}, w\right)=1\right)$ a-posteriori, using Monte-Carlo and applying the Theorem 2.2.4 of [35]. Specifically, given $\left(u^{*}, y^{*}\right)$ solving the problem (28) and for $\tilde{D}$ random samples of $w \in \mathcal{W}$, we compute the empirical number of scenarios that failed at least one constraint: $z=\sum_{i=1}^{\tilde{D}} H\left(u^{*}, w_{i}\right)$. Then, based on the Theorem 2.2.4 of [35] we can compute the confidence interval $[L(z), U(z)]$ at level $1-\tilde{\alpha} \in(0,1)$ of $\mathbb{P}\left(w \in \mathcal{W}, H\left(u^{*}, w\right)=1\right)$. One special case refers to $z>6$ and $\tilde{D}-z>6$ where the normal approximation gives:

$$
\begin{aligned}
& L(z) \approx \frac{z}{\tilde{D}}-\frac{\eta}{\tilde{D}} \sqrt{z\left(1-\frac{z}{\tilde{D}}\right)}, \\
& U(z) \approx \frac{z}{\tilde{D}}+\frac{\eta}{\tilde{D}} \sqrt{z\left(1-\frac{z}{\tilde{D}}\right)},
\end{aligned}
$$

where $\mathcal{N}_{0,1}(\eta)=\frac{\tilde{\alpha}}{2}$.

A second special case is when $z=0$, where the confidence interval of $\mathbb{P}\left(w \in \mathcal{W}, H\left(u^{*}, w\right)=1\right)$ is $[0, \tilde{U}(\tilde{D})]$ with

$$
\begin{array}{r}
\tilde{U}(\tilde{D})=1-\left(\frac{\tilde{\alpha}}{2}\right)^{\frac{1}{\tilde{D}}} \\
=\frac{1}{\tilde{D}} \log \left(\frac{2}{\tilde{\alpha}}\right)+o\left(\frac{1}{\tilde{D}}\right) \text { for large } \tilde{D} .
\end{array}
$$

If $\alpha=\tilde{\alpha}$, we characterize the scenario reduction methodology as effective if $U(z) \leq \epsilon$. In addition, if $U(z)<\epsilon$, then the upper bound on $\mathbb{P}\left(w \in \mathcal{W}, H\left(u^{*}, w\right)=1\right)$ becomes refined a-posteriori.

\section{NON-APPLICABILITY OF EXISTING RELAXATION TECHNIQUES}

The relaxation techniques used in literature, mainly relax the equality of Eq. (3) into an inequality, turning the problem into a convex one. As in this paper the battery (i.e., considered as a controllable resource) is constrained, we focus on the problem formulation of [4]. Also, for the purposes of this section, we do not consider upper bounds on voltage values and ampacity constraints (Section II-B), and we assume zero shunt capacitances for all lines, as indicated in [4]. However, even under this less restricted problem formulation compared to Section II-B, we can make the following observation.

Observation 1: The relaxation method of [4] for convexifying the AC OPF problem with constrained controllable loads is not, in general, compatible with scenario-based optimization.

The basic idea behind this observation is that, due to the inequalities that replace the equalities of Eq. (3), the optimization variables $f_{l}^{d}(t)$ could serve as slacks for the satisfaction of the rest of the constraints for the multiple scenarios. Thus, the optimal solution might be inexact, i.e., it might not satisfy Eq. (3) for every scenario as required in Section II-B. We provide an example that supports our observation using the problem formulation adopted in [4]; it is adapted to the scenario-based optimization and the notation of this paper. Let us consider a network consisting of one line $(N=1)$, only two scenarios and one time instance, thus, for the ease of presentation, we eliminate the index of time along with the battery capacity constraints. The considered problem in this example is the following:

$$
\begin{array}{r}
\min _{S, S_{B}, v, S^{D P}, f} w_{2} \sum_{d} \lambda_{d}\left|Q_{1}^{d}\right|+w_{3} \sum_{d} \lambda_{d}\left|P_{1}^{d}\right| \\
\text { s.t. } \forall d \in\{1,2\}, \\
P_{1}^{d}=p_{1}^{d}+p_{B, 1}^{d}+r_{1} f_{1}^{d}, \\
Q_{1}^{d}=q_{1}^{d}+q_{B, 1}^{d}+x_{1} f_{1}^{d}, \\
P_{1}^{1}=P_{1}^{2}=P^{D P}, \\
Q_{1}^{1}=Q_{1}^{2}=Q^{D P}, \\
v_{1}^{d}=1-2\left(r_{1} P_{1}^{d}+x_{1} Q_{1}^{d}\right)+\left\|z_{1}\right\|^{2} f_{1}^{d}, \\
f_{1}^{d}=\left\|S_{1}^{d}\right\|^{2}, \\
\left(p_{B, 1}^{+, d}\right)^{2}+\left(p_{B, 1}^{-, d}\right)^{2}+\left(q_{B, 1}^{d}\right)^{2} \leq\left(s_{B, 1}^{R}\right)^{2}, \\
v_{0}^{d}=1, \underline{v}^{2} \leq v_{1}^{d},
\end{array}
$$

where $p_{1}^{d}, q_{1}^{d}, d=\{1,2\}$ are given load quantities. We have further imposed the constraints (37)-(38) (i.e., we require that the error quantity weighted by $w_{5}$ in the objective function (16) will be zero). Note that, if we include the part of the objective function (16) weighted by $w_{5}$ instead of the constraints (37)(38), the new objective will not increase with the losses, thus the relaxation of [4] does not apply. Then, the constraints (37)(38) may render the existing relaxation techniques inexact for the scenario-based optimization, as it is explained below.

According to [4], Eq. (40) will be relaxed as $f_{1}^{d} \geq$ $\left\|S_{1}^{d}\right\|^{2}, d=\{1,2\}$, leading to a convex problem formulation. In this case, it is shown in [4] that for a single scenario, the optimal solution of the relaxed problem is an exact solution, i.e., it satisfies Eq. (40). In our example we show that for scenario-based optimization, there exists a scenario $d$ for which $f_{1}^{d *}>\left\|S^{D P *}\right\|^{2}$ (where * indicates the optimal values of the relaxed problem), and for which the optimal solution is not exact as it does not satisfy equality (40) of the non-relaxed problem. Note that, in our example, the mild conditions demanded by [4] for the exactness of the solution are satisfied for every scenario. Obtaining an exact solution by the relaxed problem means that $f_{1}^{1 *}=f_{1}^{2 *}=\left\|S^{D P *}\right\|^{2}$. This 
means that for the active power, we should compute battery power control $p_{B, 1}^{d *}, \forall d=\{1,2\}$ so that

$$
p_{B, 1}^{d *}=P^{D P *}-p_{1}^{d}-r_{1}\left\|S^{D P *}\right\|^{2},
$$

and similarly for the reactive power. However, due to the upper bounded battery power control values, the satisfaction of Eq. (43) may not be feasible for both scenarios. Then, the relaxed problem formulation uses the relaxation of the Eq. (40) leading to $f_{1}^{1^{*}}>\left\|S^{D P *}\right\|^{2}$ and/or $f_{1}^{2^{*}}>\left\|S^{D P *}\right\|^{2}$ and rendering non-exact the solution of the relaxed problem. Thus, the relaxed problem will lead to a solution, but this solution will not correspond to the one of the non-relaxed problem, contrary to the case of solving based on only one scenario.

We assign numerical values (in pu) to further illustrate our observation. Specifically, $s_{B, 1}^{R}=0.01, z_{1}=0.3458+j 0.0067$, $\underline{v}=0.9$ and $p_{1}^{1}=0.0160, p_{1}^{2}=0.0418$ with a power factor equal to 0.95 . Then, the optimal solution of the relaxed problem gives $f_{1}^{1^{*}}=0.0483, f_{1}^{2^{*}}=0.0012, P^{D P *}=0.0323$, $Q^{D P *}=0.0137$. Thus, $f_{1}^{1 *}>\left\|S^{D P *}\right\|^{2}$ whereas $f_{1}^{2 *}=$ $\left\|S^{D P *}\right\|^{2}$ due to the battery constraint of Eq. (41).

\section{The CoDistFlow SCHEME}

In this section, we propose CoDistFlow, an iterative algorithm for approximating the solution of the non-convex scenario-based AC OPF formulated in Section II-G. The algorithm leverages on the DistFlow approximation, introducing appropriate correction and approximation terms at every iteration. We call this new approximation "Improved DistFlow (iDF)". DistFlow convexifies the AC OPF by considering that losses in the lines' longitudinal-impedances defined by the quantities $r_{l} f_{l}^{d}(t)$ of Eq. (1) and $x_{l} f_{l}^{d}(t)$ of Eq. (2), are neglected (i.e., Eq. (3) is not applied), thus also misestimating the operational margins with respect to the voltage and ampacity limits. The iDF module introduces the corrections $\hat{p}_{l}^{d}(t), \hat{q}_{l}^{d}(t)$ that replace $r_{l} f_{l}^{d}(t)$ and $x_{l} f_{l}^{d}(t)$, respectively, and also $\hat{v}_{l}^{d}(t)$ used to mimic the voltage constraint of Eq. (4). Also, iDF approximates the voltage magnitudes, $\sqrt{v_{i}^{d}(t)}$, in the denominators of the ampacity constraints of Eqs. (7)-(10) by constants $\tilde{v}_{i}^{d}(t)$, overall keeping the problem convex. The values of the corrections and approximations are obtained by solving load flow (LF) problems.

CoDistFlow operates as follows (see Algorithm 1). At iteration $k$, iDF (Section IV-A) takes as inputs (i) the corrections terms for losses and voltages $C$, whose $j$-th element is $C_{j}$, (ii) the approximations for voltages $\tilde{V}$ and (iii) the constant load injections $s(t, d)$, with

$$
\begin{aligned}
C(t, d) & =\left[\hat{P}^{d}(t) ; \hat{Q}^{d}(t) ; \hat{V}^{d}(t)\right], \\
s(t, d) & =\left[p^{d}(t) ; q^{d}(t)\right],
\end{aligned}
$$

and outputs (i) the optimal dispatch-plan at the PCC, $S^{D P}$, (ii) the electrical state of the grid $E^{\prime}$ and (iii) the battery power trajectories $s_{B}$, with

$$
\begin{aligned}
E^{\prime}(t, d) & =\left[P^{d}(t) ; Q^{d}(t) ; v^{d}(t)\right], \\
s_{B}(t, d) & =\left[p_{B}^{d}(t) ; q_{B}^{d}(t)\right] .
\end{aligned}
$$

Then, new correction and approximation terms are computed by solving a full AC load flow, taking into account the newly computed battery trajectories. The algorithm terminates when the variations of the correction terms, of the voltage approximations and of the battery trajectories between the last two consecutive iterations are smaller than an error tolerance $\epsilon_{c}>0, \epsilon_{v}>0$, and $\epsilon_{B}>0$, correspondingly. Note that, $s(t, d)$ is considered known and constant for each time $t$ and scenario $d$. Also, we differentiate between the electrical states derived by iDF and LF by utilizing a ' symbol for the former. When the algorithm terminates, the obtained solution satisfies the exact (AC) power flow equations and the exact operational constraints within the tolerance bounds imposed by the convergence criterion (see also Theorem 2).

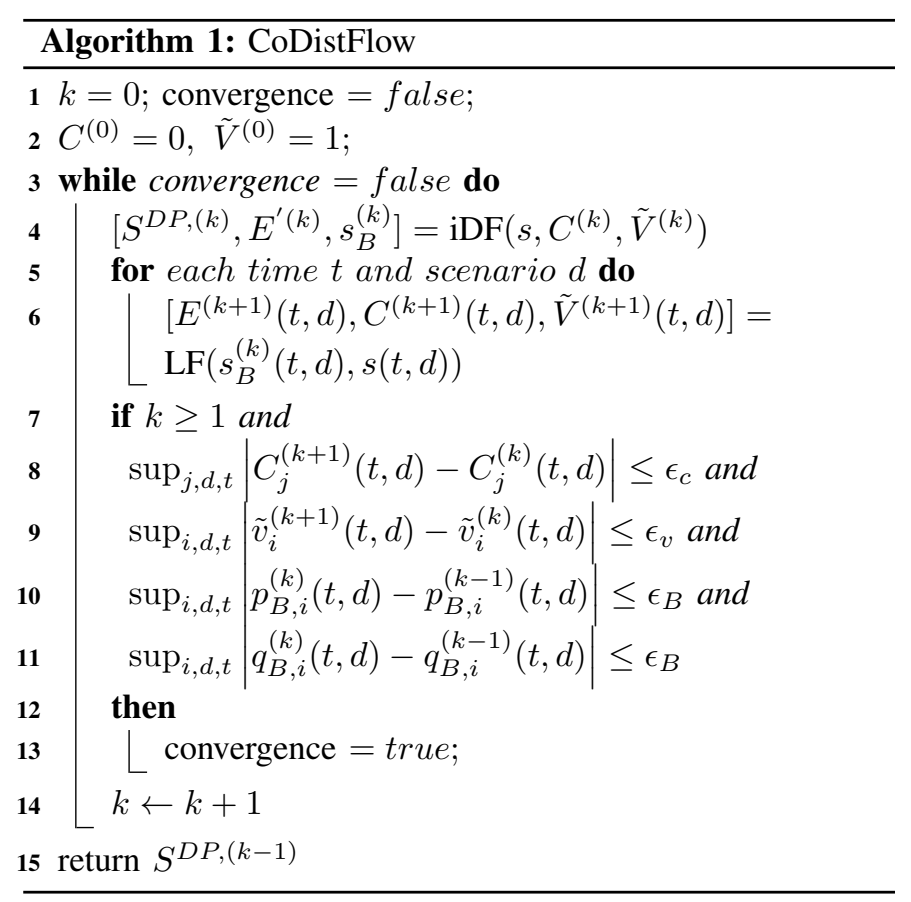

\section{A. Improved DistFlow (iDF) Module}

The iDF module is given as $\left[S^{D P}, E^{\prime}, s_{B}\right]=\operatorname{iDF}(s, C, \tilde{V})$. It solves a similar optimization problem as Section II-G, but with the following differences. First, it applies the DistFlow approximation, and second it corrects the DistFlow approximation via the correction and approximation terms, as previously described in the introductory part of this section. Therefore, 
the convex scenario-based AC OPF solved by iDF is:

$$
\begin{array}{r}
\min _{S, S_{B}, v, S^{D P}}(25), \quad s . t . \forall t, d, l, \\
(5),(10)-(13),(17)-(22), \\
v_{l}^{d}(t)=v_{\mathrm{up}(l)}^{d}(t)-2 \Re\left\{z_{l}^{*}\left(S_{l}^{d}(t)+j v_{\mathrm{up}(l)}^{d}(t) \frac{b_{l}}{2}\right)\right\} \\
+\hat{v}_{l}^{d}(t), \\
P_{l}^{d}(t)=\sum_{k: \mathbf{G}_{l k}=1} P_{k}^{d}(t)+p_{l}^{d}(t)+p_{B, l}^{d}(t)+\hat{p}_{l}^{d}(t), \\
Q_{l}^{d}(t)=\sum_{k: \mathbf{G}_{l k}=1} Q_{k}^{d}(t)+q_{l}^{d}(t)+q_{B, l}^{d}(t) \\
-\left(v_{\mathrm{up}(l)}^{d}(t)+v_{l}^{d}(t)\right) b_{l} / 2+\hat{q}_{l}^{d}(t), \\
\Re\left\{i_{l}^{d}(t)\right\}=\frac{P_{l}^{d}(t)}{\tilde{v}_{\mathrm{up}(l)}^{d}(t)}, \quad \Im\left\{i_{l}^{d}(t)\right\}=\frac{Q_{l}^{d}(t)}{\tilde{v}_{\mathrm{up}(l)}^{d}(t)}, \\
\Re\left\{i_{l}^{b, d}(t)\right\}=\left(P_{l}^{d}(t)-\hat{p}_{l}^{d}(t)\right) / \tilde{v}_{l}^{d}(t), \\
\Im\left\{i_{l}^{b, d}(t)\right\}=\Im\left\{i_{l}^{d}(t)\right\} \\
+\left(v_{\mathrm{up}(l)}^{d}(t)+v_{l}^{d}(t)\right) \frac{b_{l}}{2 \tilde{v}_{l}^{d}(t)}-\frac{\hat{q}_{l}^{d}(t)}{\tilde{v}_{l}^{d}(t)} .
\end{array}
$$

The voltage constraint of Eq. (50) derives form Eq. (4), by replacing the quantities $\left\|z_{l}\right\|^{2} f_{l}^{d}(t)$ with the corrections $\hat{v}_{l}^{d}(t), \forall t, d, l$. The power flow constraints of iDF, Eqs. (51)(52) and the ampacity constraints Eqs. (53)-(55), derive from Eqs. (1)-(2) and Eqs. (7)-(9) respectively, by replacing the quantities $r_{l} f_{l}^{d}(t), x_{l} f_{l}^{d}(t)$ with the corrections $\hat{p}_{l}^{d}(t), \hat{q}_{l}^{d}(t)$ and using the approximations $\tilde{v}_{i}^{d}(t)$ for the voltage magnitudes. Therefore, in the convex scenario-based OPF solved by iDF, the active and reactive power losses excluded in the DistFlow approximation are treated as loads at the bottom node of each line, but they are also used to compute the necessary corrections for voltages and currents.

\section{B. Load Flow (LF) Module}

The LF module is given as $[E(t, d), C(t, d), \tilde{V}(t, d)]=$ $\operatorname{LF}\left(s_{B}(t, d), s(t, d)\right)$, i.e., it computes for a specific scenario $d$ and time $t$, (i) the electrical state of the grid, (ii) the active and reactive power losses and voltage corrections and (iii) the voltage magnitude approximations. The second output is not accounted for in DistFlow and it is applied as input to the iDF as described above. The inputs of the LF include the power injections (PQ-load buses) that are given for every time and scenario. Also, we consider the batteries as PQ buses with injections computed by the iDF module at the previous iteration. Given the power injections at the buses, LF solves for a specific time and scenario, the exact power flow equations (Eqs. (1)-(4)). For the latter solution, LF applies the Newton Raphson numerical method. The existence and uniqueness of the LF solution for radial distribution networks is shown in [36] for feasible voltage magnitudes (close to 1) and in [37] for bounded voltage magnitudes. The outputs (ii) and (iii) of $\mathrm{LF}, \forall i, l, d, t$, are computed as

$$
\begin{aligned}
{\left[\hat{p}_{l}^{d}(t), \hat{q}_{l}^{d}(t), \hat{v}_{l}^{d}(t)\right] } & =f_{l}^{d}(t) \cdot\left[r_{l}, x_{l},\left\|z_{l}\right\|^{2}\right], \\
\tilde{v}_{i}^{d}(t) & =\sqrt{v_{i}^{d}(t)} .
\end{aligned}
$$

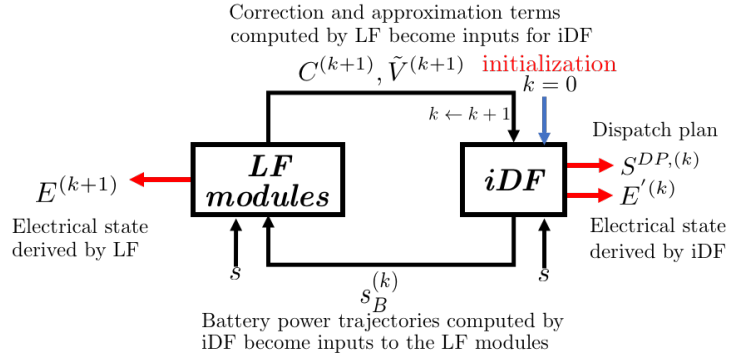

Fig. 4: Illustration of the CoDistFlow iterations.

\section{Correctness of the Solution of CoDistFlow}

From numerical evaluations, we observed that CoDistFlow converges in all cases in few iterations. This is intuitively expected, because CoDistFlow is initialized by DistFlow and iterates over DistFlow which is already a good approximation of the AC power flow equations. In Fig. 4, we illustrate the iterations of CoDistFlow and the quantities exchanged between iDF and LF. CoDistFlow converges when these exchanged quantities become stationary and we say that their values are a fixed point of CoDistFlow:

Definition 1: $\left(s_{B}^{*}, C^{*}, \tilde{V}^{*}\right)$ is a fixed point of CoDistFlow if:

1) $\left[S^{D P}, E^{\prime}, s_{B}^{*}\right]=\operatorname{iDF}\left(s, C^{*}, \tilde{V}^{*}\right)$, for some $S^{D P}, E^{\prime}$ and

2) $\left[E(t, d), C^{*}(t, d), \tilde{V}^{*}(t, d)\right]=\operatorname{LF}\left(s_{B}^{*}(t, d), s(t, d)\right)$, $\forall t, d$, for some $E$.

Since iDF (Eqs. (48)-(55)) uses external correction and approximation terms in the power flow equations, it is not entirely obvious that, at a fixed point, the exact power flow equations are satisfied. In other words, the question is whether after convergence of CoDistFlow (in the limit), $E$ and $E^{\prime}$ shown in Fig. 4 coincide. In the next theorem, we prove that it is indeed correct assuming a mild condition on the admittance matrix. It follows that, at a fixed point of CoDistFlow, the electrical state, $E^{\prime}(t, d), \forall t, d$, which is used to compute the dispatch plan $S^{D P}$, is a solution of the exact power flow equations. Furthermore, since the voltage approximations $\tilde{V}(t, d), \forall t, d$ are computed based on $E(t, d), \forall t, d$, at the fixed point, they are exact and the exact ampacity constraints in Eqs. (7)-(10) are satisfied by the solution of CoDistFlow.

Let us define the matrices $\mathbf{H}=(\mathbf{I}-\mathbf{G})^{-1}$ and $\mathbf{M}=$ $\operatorname{diag}(x) \mathbf{H} \operatorname{diag}(b)$.

Theorem 2: Assume that $\left\|\mathbf{H}^{T} \mathbf{M}\right\|_{1}<1$. Let $\left(s_{B}^{*}, C^{*}, \tilde{V}^{*}\right)$ be a fixed point of CoDistFlow, namely $\left[S^{D P}, E^{\prime}, s_{B}^{*}\right]=\operatorname{iDF}\left(s, C^{*}, \tilde{V}\right)$, $\left[E(t, d), C^{*}(t, d), \tilde{V}^{*}(t, d)\right]=\operatorname{LF}\left(s_{B}^{*}(t, d), s(t, d)\right), \forall t, d$. Then $E^{\prime}=E$, i.e., the electrical states given by CoDistFlow and LF coincide.

A sufficient condition for $\left\|\mathbf{H}^{T} \mathbf{M}\right\|_{1}<1$ is [7]:

$$
\max _{\forall l} x_{l} \max _{\forall i} b_{i} \leq \frac{1}{N^{2}} \text {. }
$$

The proof is given in Appendix A.

\section{EVALUATION RESUlts}

In this section, we evaluate CoDistFlow in two cases, (i) a 4bus network (see Fig. 5a) and (ii) in a real Swiss distribution 


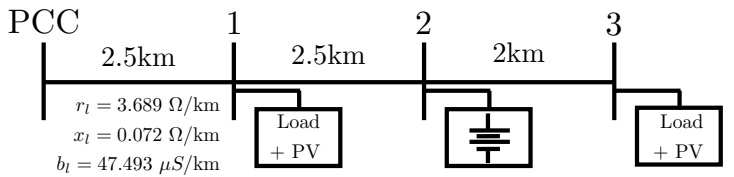

(a) Single-line diagram of the 4-bus network.

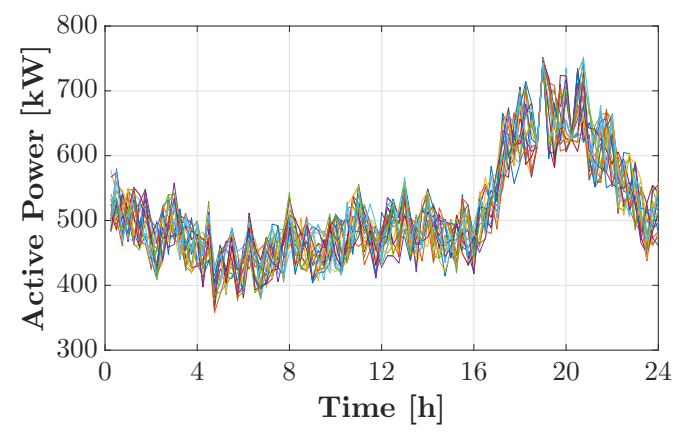

(b) Prosumption scenarios at nodes 1 and 3 after scenario reduction. Reactive power corresponds to a power factor 0.95 .

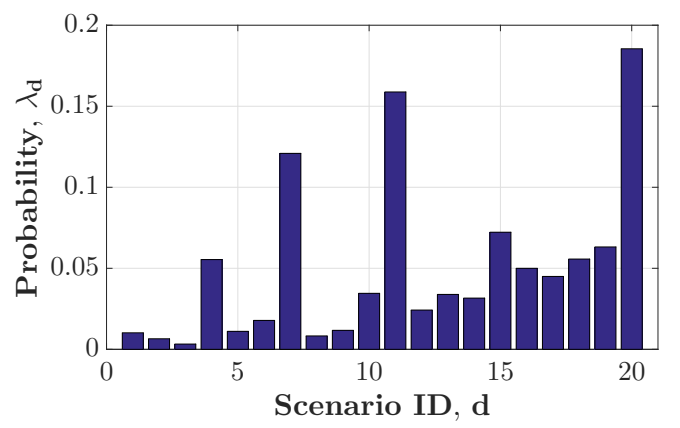

(c) Probabilities of prosumption scenarios at nodes 1 and 3 after scenario reduction.

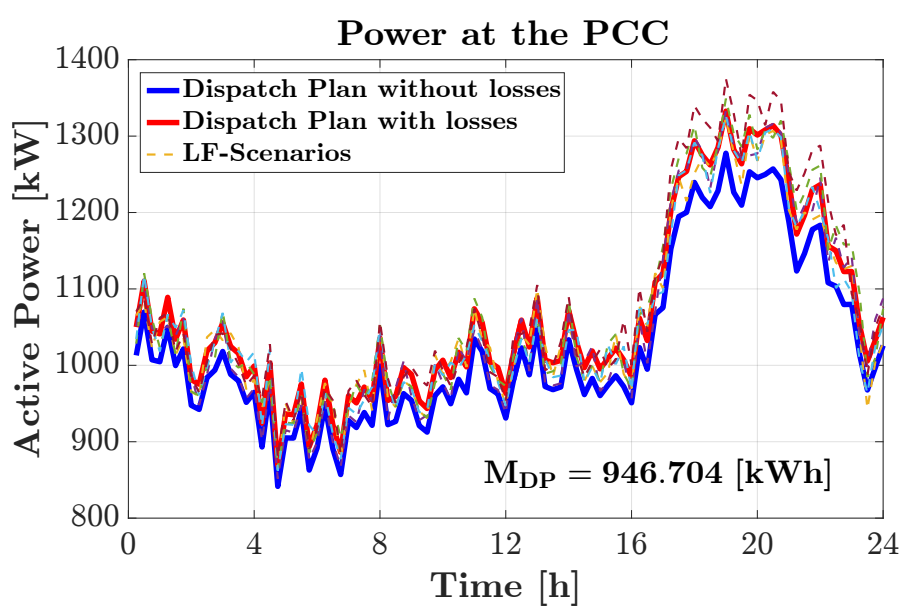

(d) Comparison of dispatch plans. Efficiency battery model.

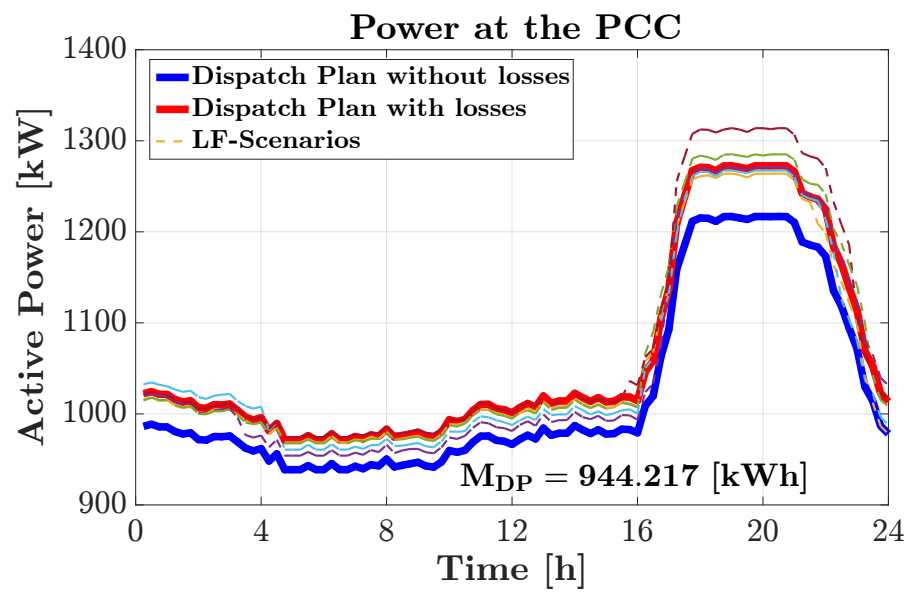

(e) Comparison of dispatch plans. Resistance battery model.

Fig. 5: Day-ahead results for the 4-bus network. We consider $w_{1}=0.0005, w_{3}=1, w_{5}=10, w_{7}=0.001$.

grid. The former is used to show the effect of the grid and battery losses and of the battery model on the optimal dispatch plan, along with constituting a minimum test-case that can be easily reproducible. Indeed, for the 4-bus network we provide comparative results with an iterative linearization algorithm inspired by similar approaches from the existing literature. The latter case refers to a larger distribution grid and serves as a more realistic case study of the effect of the grid and battery losses on the effectiveness of the optimal dispatch plan.

CoDistFlow converged in 3 iterations for the 4-bus network and in 4 iterations for the real grid. We did not observe convergence issues in all the simulations we ran. However, as the computation is performed day-ahead, even a larger number of iterations until convergence is acceptable. We consider $\underline{v}=0.9$ $\mathrm{pu}, \bar{v}=1.1 \mathrm{pu}, w_{2}=w_{4}=w_{6}=1, \underline{\mathrm{E}}_{B, i}=0.15 \overline{\mathrm{SoE}}_{B, i}$, $\overline{\mathrm{E}}_{B, i}=0.85 \overline{\operatorname{SoE}}_{B, i}, \forall i, \operatorname{SoE}_{B, i}^{d}(0)=0.3 \overline{\operatorname{SoE}}_{B, i}, \forall i, d$ and $a_{B}=0.1, \Delta t=0.25 \mathrm{~h}$.

The choice of the scenarios is performed as follows. We fix an upper bound of the probability of constraint violation by the optimal solution at a random realization of the uncertain parameters equal to $\epsilon=3 \%$ with confidence level $1-\alpha=99 \%$ (see Section II-H). Note that, this upper bound refers to the violation of any single constraint that may not lead to the inoperability of the grid. Then, according to Eq. (30), the number of scenarios should be $D \approx 55,000$. For each of the above evaluation cases, we utilize a prosumption forecast over one day and we create random variations around it (at most $10 \%$ above and below) via Monte-Carlo, producing 55,000 scenarios in total. Afterwards, we perform scenario reduction as described in Section II-H. After solving the scenario-based $\mathrm{AC} \mathrm{OPF}$ we perform a-posteriori calculations to evaluate the effectiveness of the scenario reduction as described in Section II-H, and we refine the upper bound $\epsilon$. In each specific evaluation case, we provide more details on the used prosumption forecast and we illustrate the scenarios obtained after the reduction.

\section{A. Case 1}

The resistance of the battery model is $0.017 \Omega$, obtained as mentioned in Section II-E2. Each line has an ampacity limit of 80 A. The battery characteristics are $s_{B, 2}^{R}=720 \mathrm{~kW}$, $\overline{\mathrm{SoE}}_{B, 2}=500 \mathrm{kWh}$. Based on the grid's parameters given in 


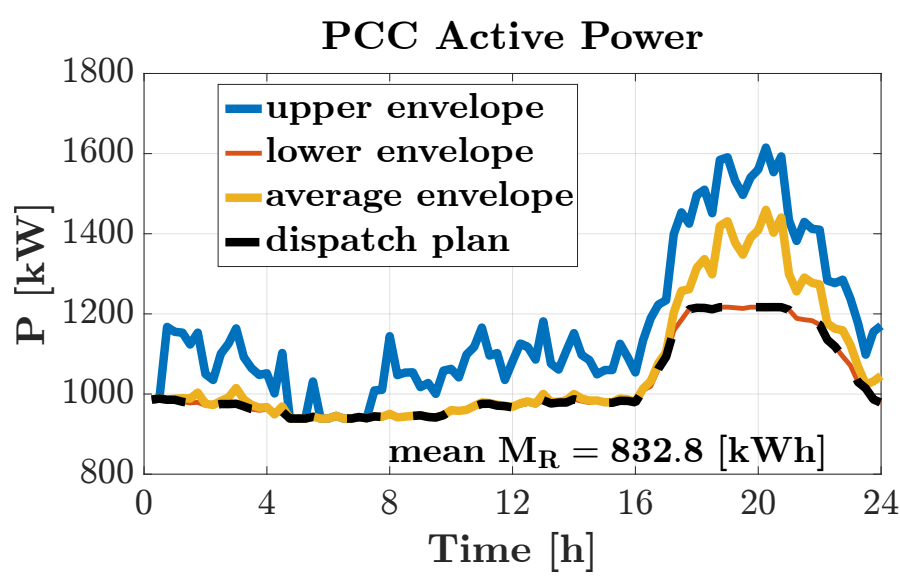

(a) Dispatch plan vs. actual realization at the PCC. DistFlow approximation.

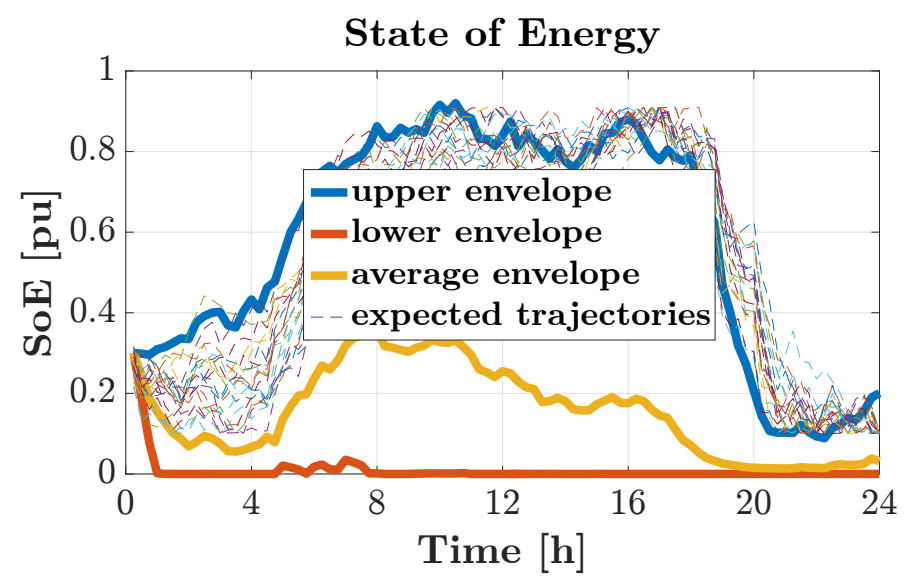

(b) Battery trajectories for DistFlow approximation

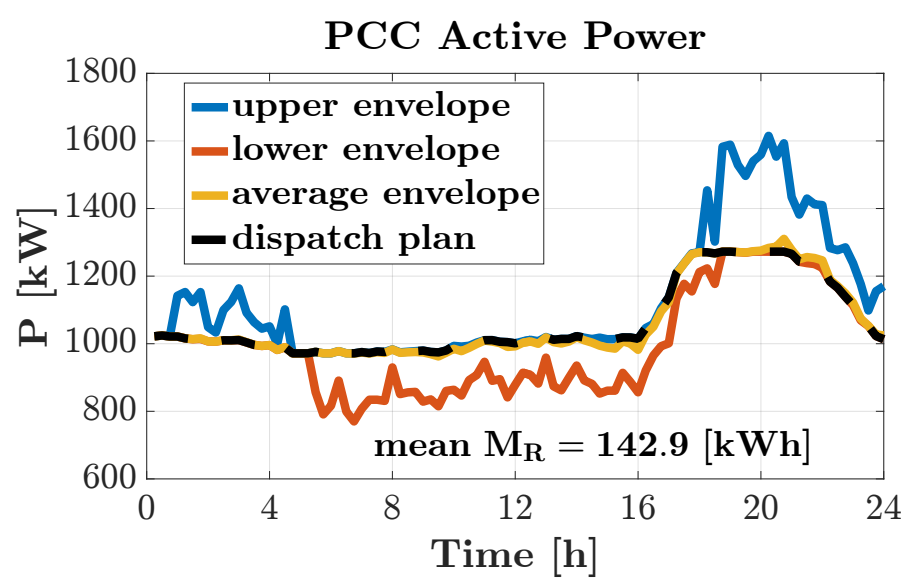

(c) Dispatch plan vs. actual realization at the PCC. Proposed CoDistFlow.

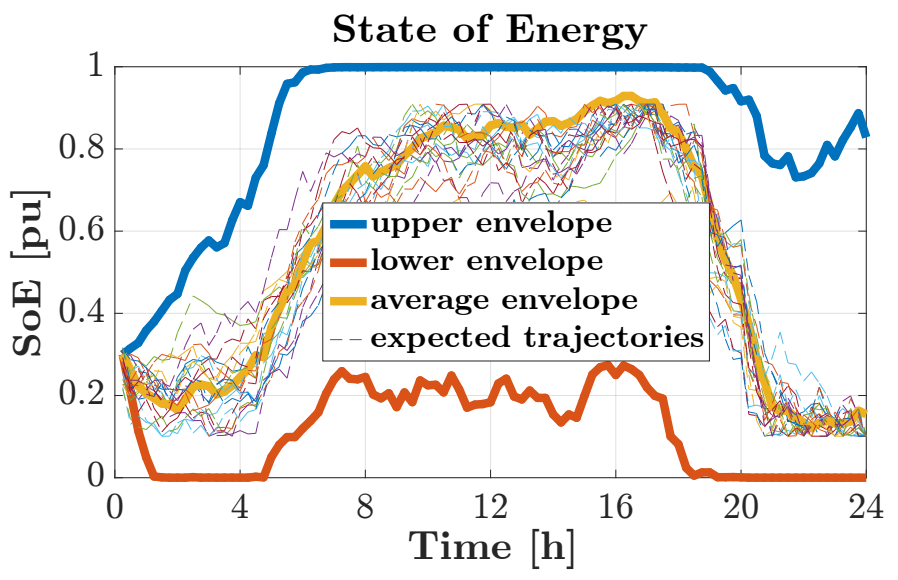

(d) Battery trajectories for CoDistFlow.

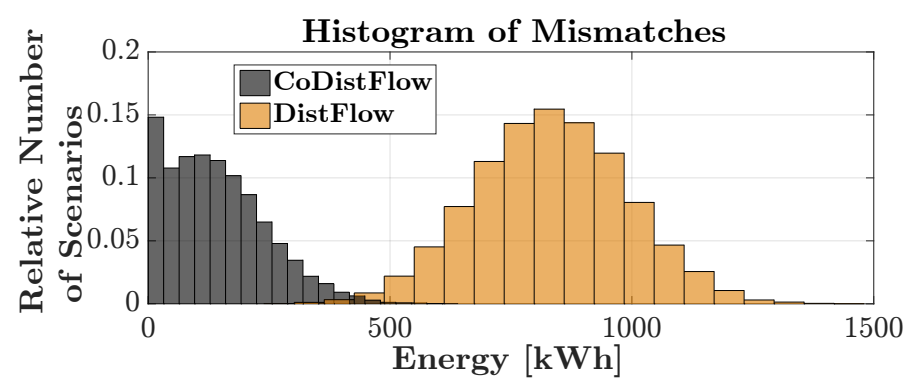

(e) Histogram of mismatches, $M_{R}$, for all scenarios.

Fig. 6: Monte-Carlo simulation results for the 4-bus network.

Fig. 5a, this grid satisfies the sufficient condition of Eq. (58) in Theorem 2. After scenario reduction, we consider a set of 20 scenarios for the prosumption of nodes 1 and 3 (see Fig. 5b), that are based on a forecast for a winter day. The probability density of the considered scenarios is illustrated in Fig. 5c.

In Figs. 5d and 5e, we compare the dispatch plan using the DistFlow approximation and the CoDistFlow scheme for the two battery models, namely, the traditional model with the efficiencies and the model with the resistance. We define $M_{D P}$ as the sum for the whole day of the absolute differences between the dispatch plans of DistFlow and CoDistFlow. Firstly, we observe that $M_{D P}$ is more than $900 \mathrm{kWh}$ for both battery models and it is due to the grid losses. Intuitively, in case of DistFlow the battery can experience problems to dispatch since these losses are larger than the battery capacity. Secondly, we observe that the battery model impacts the shape of the dispatch plan, and specifically the battery model with the resistance leads to a much smoother dispatch plan.

The dashed lines in Figs. 5d, 5e correspond to the power at the PCC, $P_{1}^{d}($.$) , for several scenarios d$, computed by the LF using as input the battery power values obtained by CoDistFlow after convergence. They aim at verifying that the output of CoDistFlow satisfies the LF equations, i.e., to support numerically Theorem 2 . Their differences from the dispatch plan are explained by the error weighted by $w_{5}$ in the objective. When computing the dispatch plans, the power 
factor at the PCC is always equal to 1, thus satisfying for both DistFlow and CoDistFlow the hard constraint of Eq. (6) with $\cos (\phi)_{\min }=0.95$, for all time intervals, all scenarios and for both battery models.

We evaluated the derived dispatch plans in Fig. 5e for 16,000 scenarios created via Monte-Carlo in the way that is described above for the construction of the initial set of scenarios. In other words, we aim at examining if random scenarios that do not fall in the initial set of the 55,000 scenarios can follow the dispatch plans of DistFlow and CoDistFlow and if they satisfy the constraints of Eq. (27). Firstly, all scenarios satisfied the constraints; thus, we can apply Eq. (33), for computing the confidence interval of the probability that the optimal solution at a random scenario (within the uncertainty model considered above) will violate any single constraint. The result is that, at level of $99 \%$, this probability lies in $[0,0.0003]$, i.e., the probability is much less than $3 \%$, as obtained above via the number of scenarios considered in the scenario-based day-ahead problem (before scenario reduction). We note that, also in this case, the power factor is equal to 1 for all random scenarios, satisfying the hard power factor constraint of Eq. (6).

In Figs. 6a, 6b (DistFlow) and 6c, 6d (CoDistFlow), we compare the actual realization at the PCC with the corresponding dispatch plan and show the actual battery SoE trajectories compared to the expected ones, where the latter are computed day-ahead for the scenarios at the reduced set. The lower/upper/average envelope takes for each time the minimum/maximum/average value for all scenarios, respectively. The realized (actual) battery SoE trajectories are computed by the TTC model [3], considered as capable to reproduce the true battery behavior. We define $M_{R}$ as the daily sum of the absolute differences between the actual realization at the PCC and the dispatch plan for a specific scenario. We observe that, if not accounting for the grid and battery losses (Fig. 6a), the mean value of $M_{R}$ is more than 5.5 times bigger compared with Fig. 6c. Indeed, in Fig. 6a the battery cannot handle the overall losses that is verified by the increased actual value at the PCC compared with the dispatch plan for all scenarios (i.e., the lower envelope lies on the dispatch plan). This is the reason why the actual battery trajectories fell below the expected ones in Fig. 6b (i.e., the average envelope lies much lower the expected trajectories). On the contrary, the mismatches in Fig. 6c emerge both above and below the dispatched power at the PCC, thus indicating that their cause lies in random variations of the prosumption not accounted in the considered scenarios. This is aligned with the actual battery trajectories in Fig. 6d, where their mean envelope almost follows the expected ones computed in day-ahead.

In Fig. 6e, we compare the histogram of the mismatches, $M_{R}$, for all the scenarios between DistFlow and CoDistFlow. We observe that, the histogram of the former is biased towards very high values given that overall losses are not considered in the dispatch plan. On the contrary, the mismatch values in CoDistFlow are much closer to zero, though they may deviate from zero due (i) to the difference of the prosumption in the actual realization with respect to the scenarios used for computing the dispatch plan, (ii) to the fact that the dispatch plan does not exactly correspond to the power at the PCC for all considered scenarios (error quantity weighted by $w_{5}$ ), or (iii) to the fact that in the actual realization we apply a more precise battery model (TTC).

\section{B. Comparative Evaluation Results in Case 1}

In this part we compare CoDistFlow with an iterative algorithm for solving the scenario-based AC OPF which is similar with the iterative linearization method proposed in [13]. The details of this algorithm, denoted as iL-OPF (iterative linearized OPF), are provided in Appendix B.

In Fig. 7a, we compare the dispatch plans obtained by DistFlow, CoDistFlow and iL-OPF. For comparison purposes, we initialize iL-OPF using the optimal battery trajectories given by DistFlow (i.e., by the first iteration of CoDistFlow). The initial voltages, currents and power at the PCC for every scenario and time are given by LF using as inputs the same battery trajectories and the fixed load injections for each scenario. As a result, the grid and battery losses corresponding to the optimal battery trajectories given by DistFlow and the fixed load injections for each scenario are reflected in the initial voltages, currents and power at the PCC. CoDistFlow has the advantage of being initialized always by DistFlow and therefore there is no ambiguity on its initialization as it is the case for iL-OPF. CoDistFlow converged after 3 iterations and iL-OPF after more than 150 iterations, while the dispatch plans obtained by both CoDistFlow and DistFlow are much smoother than the one obtained via iL-OPF.

Following a similar procedure as in Section V-A, we computed the mismatch, $M_{R}$, between the actual realization at the PCC and the dispatch plan, for DistFlow, CoDistFlow and iL-OPF, for 200 random scenarios. Via this procedure, we basically compare the effectiveness of the dispatch plans (Fig. 7a). The results are shown in Fig. 7b, where it is clearly observed that iL-OPF leads to significantly higher mismatch values than both DistFlow and CoDistFlow. This can be intuitively explained by the iterative linearization of the $\mathrm{AC}$ power flow equations around suboptimal operating points. In general, comparing CoDistFlow with iL-OPF, we can state that CoDistFlow is a more stable algorithm in terms of its convergence (i.e., the initial conditions are specific and lead to convergence within a few iterations), while showing low mismatch values.

\section{Case 2}

The realistic case 2 consists of 34 buses. Fig. 8a presents the 6 scenarios for the PV generation at node 22 (that lies far from the PCC). These scenarios derive from the scenario reduction as described above based on the forecast for a summer day. The battery is connected to node 2 (next to the PCC), with $6 \mathrm{MW}$ and $6 \mathrm{MWh}$ as nominal power and energy values. The $\mathrm{max} / \mathrm{min}$ (magnitude) line impedance is $(0.34+j 0.24) /(0.025+j 0.01) \Omega$, the $\max / \mathrm{min}$ shunt capacitance is $200.15 / 6.73 \mu \mathrm{S}$ and the $\mathrm{max} / \mathrm{min}$ ampacity limit is $400 / 140$ A. Note that, based on these parameter values, this grid satisfies the sufficient condition of Eq. (58) in Theorem 2. As the grid has a larger scale, the battery model is not expected 


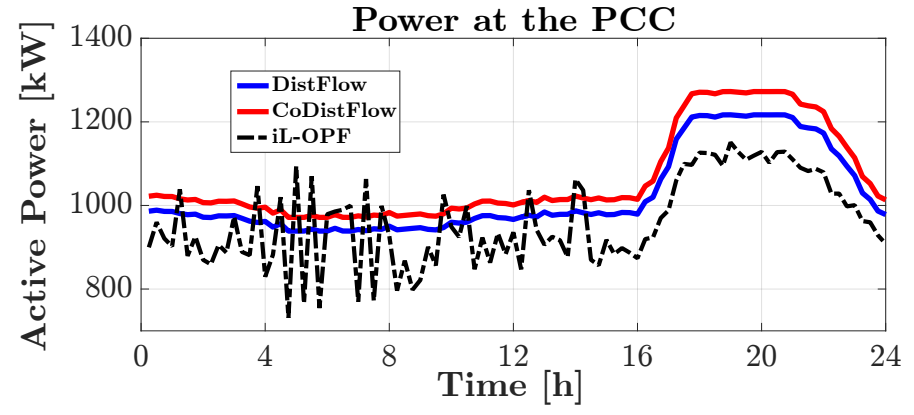

(a) Comparison of dispatch plans.

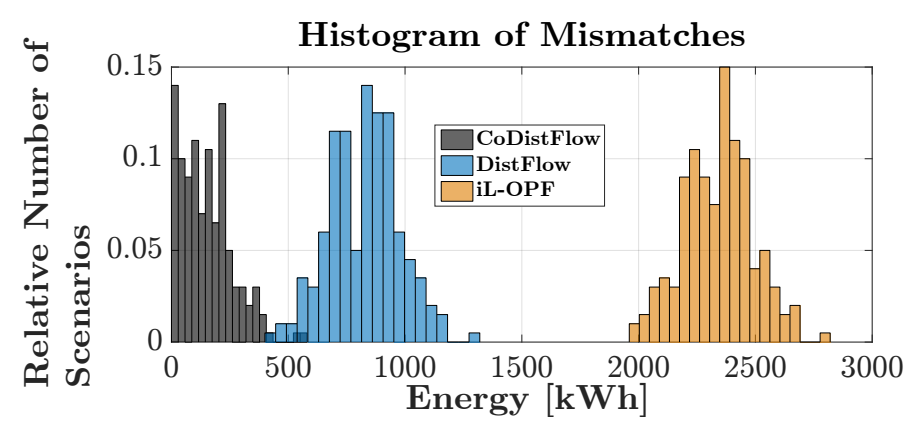

(b) Histogram of mismatches, $M_{R}$, for all scenarios.

Fig. 7: Comparison of DistFlow, CoDistFlow and iL-OPF.

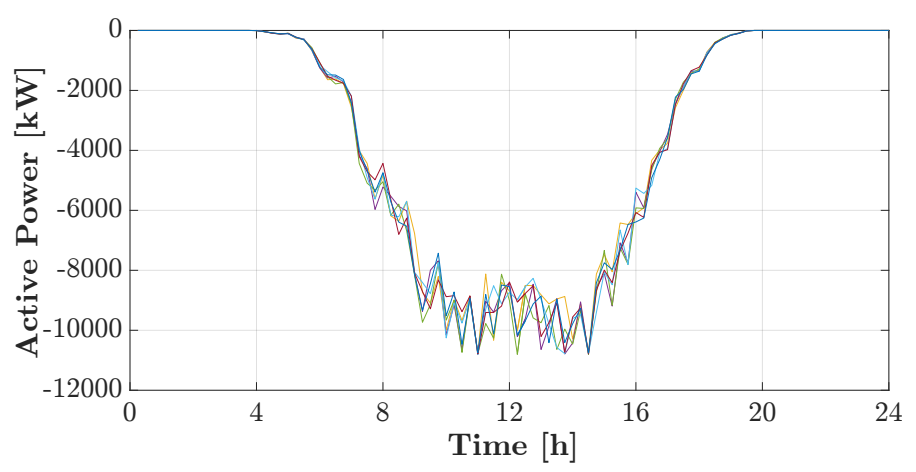

(a) PV injection scenarios at node 22 after scenario reduction. The probabilities of the scenarios are: $\lambda_{1}=0.059, \lambda_{2}=0.0568, \lambda_{3}=0.1087, \lambda_{4}=0.1895$, $\lambda_{5}=0.1172 \lambda_{6}=0.4688$.

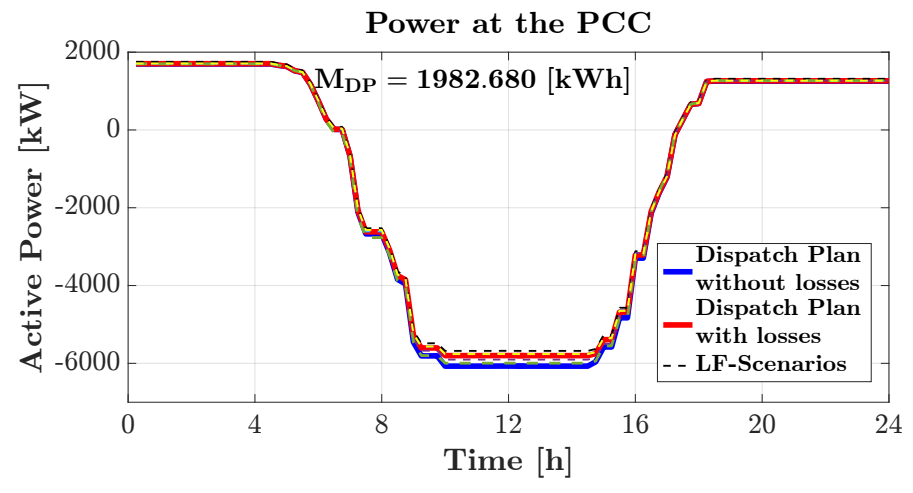

(b) Comparison of dispatch plans.

Fig. 8: Day-ahead results for the real Swiss distribution grid. We consider $w_{1}=1, w_{3}=0 w_{5}=100, w_{7}=0.0001$.

to affect the dispatch plan (Section II-E), as it is also observed in our simulations. Thus, we present only the results derived using the battery model with the resistance.

Fig. 8b compares the dispatch plan computed via DistFlow and CoDistFlow. The mismatch $M_{D P}$ attains a value around $2 \mathrm{MWh}$ that can be characterized as large compared with the battery capacity ( $6 \mathrm{MWh})$. When computing the dispatch plans, the power factor at the PCC satisfies for all time intervals and all scenarios considered in the scenario-based, day-ahead optimization problem the hard constraint of Eq. (6), with $\cos (\phi)_{\min }=0.95$.

Similarly to Case 1 , we evaluated the derived dispatch plans in Fig. $8 \mathrm{~b}$ for 10,000 scenarios created via Monte-Carlo. In the a-posteriori assessment, all the scenarios satisfied the constraints, thus using Eq. (33), the confidence interval of the probability that the optimal solution at a random scenario will violate any single constraint, at confidence level $99 \%$, is equal to $[0,0.0005]$.

In Figs. 9a, 9b (DistFlow) and 9c, 9d (CoDistFlow), we compare the actual realization at the PCC with the dispatch plan and we show the actual battery SoE trajectories compared to the expected ones (computed day-ahead). We observe that, if not accounting for grid and battery losses (Fig. 9a), the average mismatch between the dispatch plan and the actual realization,
$M_{R}$, is more than 6 times bigger, compared with Fig. 9c, as in Fig. 9a the battery cannot handle the grid and battery losses. The latter is illustrated by (i) in Fig. 9a, the lower envelope lies on the dispatch plan and (ii) in Fig. 9b, the actual battery SoE trajectories fell on average below the expected ones. On the contrary, the mismatches in Fig. 9c emerge both above and below the dispatched power at the PCC and in Fig. 9d the average envelope of the battery trajectories almost follows the expected ones computed in day-ahead.

Finally, in Fig. 9e, we compare the histogram of the mismatches, $M_{R}$, for all the scenarios between DistFlow and CoDistFlow. Similarly, to Case 1, the histogram of the former is biased towards very high values given that the overall losses are not considered in the dispatch plan. The non-zero mismatch observed for CoDistFlow is not attributed to the overall losses rather to the same reasons explained in Section V-A.

The economic impact of applying the CoDistFlow scheme rather than the DistFlow approximation can be evaluated by considering the difference in the amount of reserve which is activated in each respective case and the price of reserve per unit of activated regulating power. We assume the dispatched distribution network as a price taker and we consider average figures of the regulating power prices in the NordPool electric- 


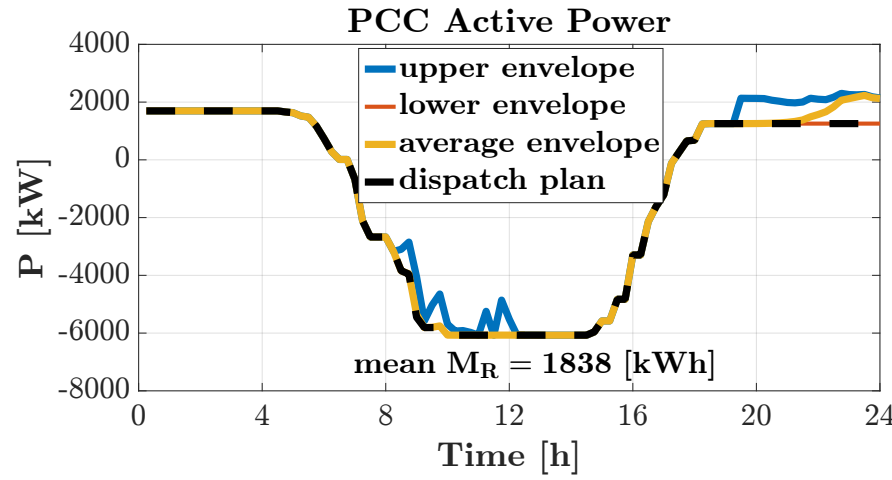

(a) Dispatch plan vs. actual realization at the PCC. DistFlow approximation.

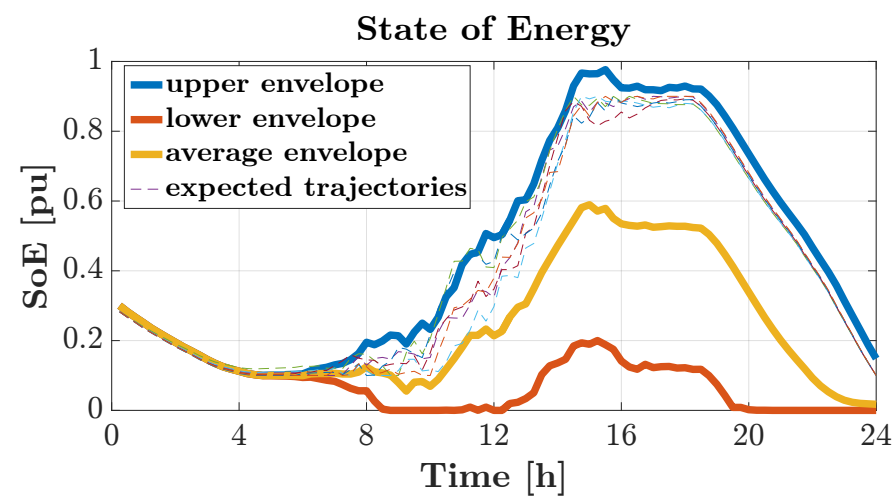

(b) Battery trajectories for DistFlow approximation.

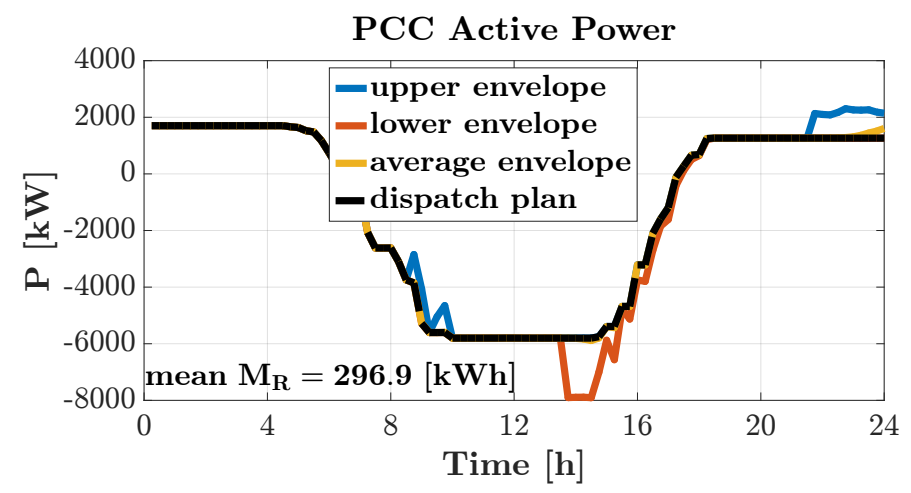

(c) Dispatch plan vs. actual realization at the PCC. Proposed CoDistFlow.

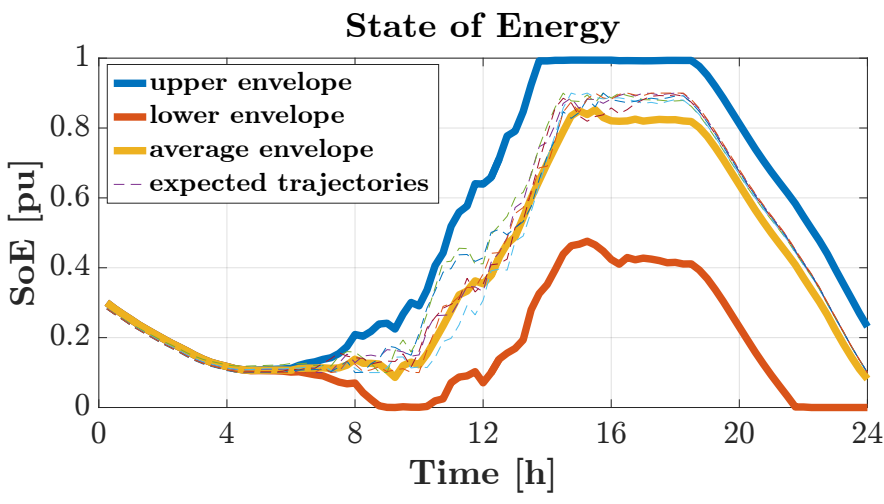

(d) Battery trajectories for CoDistFlow.

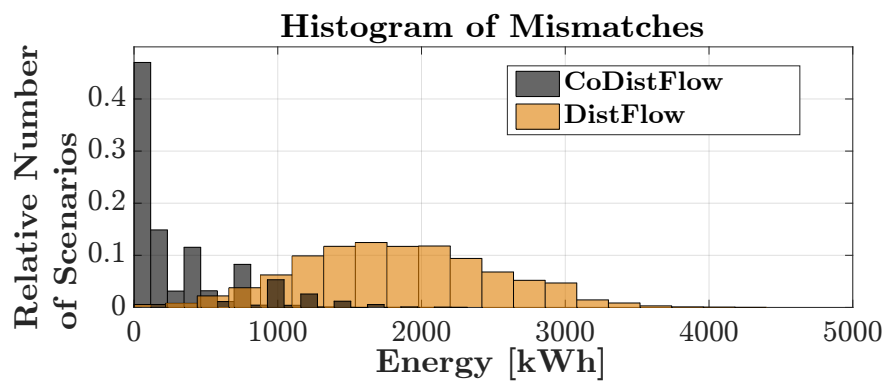

(e) Histogram of mismatches, $M_{R}$, for all scenarios.

Fig. 9: Monte-Carlo simulation results for the real Swiss distribution grid.

ity market (29.23 EUR per MWh derived as the mean of the prices 26.02 and 32.44 EUR per MWh for down and up regulation, respectively ${ }^{3}$ ). Then, the economic loss for not following the dispatch plan is equal to $1.838 \cdot 29.23=53.85$ EUR per day (using the mean $M_{R}$ value in Fig. 9a), when dispatching the operation of the feeder with the DistFlow approximation, while it is decreased to $0.2969 \cdot 29.23=8.7$ EUR per day (using the mean $M_{R}$ value in Fig. 9c) when the CoDistFlow scheme is used.

\section{Discussion on the Extension to Multiphase Unbalanced GRIDS AND TO MEShed GRIDS}

In this section, we discuss a potential way to extend CoDistFlow to unbalanced distribution grids and to meshed

\footnotetext{
${ }^{3}$ Average hourly regulating power prices for DK2 for 2016 (http://www.nordpoolspot.com/).
}

distribution grids. However, these extensions are non-trivial processes and they constitute part of our future research.

Regarding the extension of CoDistFlow to unbalanced grids, let us consider radial power grids whose generic components connected between two of their buses are characterized by circulant shunt admittance and longitudinal impedance matrices (i.e., for a matrix of rank $n$, its eigenvectors are composed by the roots of unity of order $n$ ). For these grids, it is possible to decompose all the nodal/flow voltages, currents and powers with the well-known sequence (or symmetrical components) transformation. The result of the sequence transformation is composed by three symmetrical and balanced three-phase circuits for which the proposed CoDistFlow can be applied as is. The main problem of this approach, however, is given by the transformation of the voltage/current constraints from the phase domain to the corresponding ones in the 
sequence domain. Indeed, such a transformation couples the voltage/currents constraints in the sequence domain. However, it is possible to separately bound the zero and negative sequence terms of nodal voltage magnitude and lines current flows by using more conservative constraints as the magnitude of these quantities are restricted by standards/norms (i.e., their maximum magnitudes are known a-priori). The binding of the zero and negative sequences associated with the voltages and currents should allow to decouple the positive sequence. Then, we can apply the proposed CoDistFlow process to the three sequences for which we may derive different voltage/current inequalities. Once the three problems are solved, we can transform the obtained voltage/currents/powers in the sequence domain back to the (unbalanced) phase domain.

Regarding the extension to meshed grids, note first that the proposed method relies on (i) the solution of a LF problem that, by construction, can only ensure a unique solution for radial systems and (ii) the DistFlow approximation, which also works properly only in radial systems. A straight solution would be to first "radialize" the grid (e.g., as in [38]) and apply CoDistFlow as is. Another possible solution would be to combine the grid radialization iterations [38] with the CoDistFlow iterations. Yet, as we focus on distribution networks, the method can be applied to most of actual systems that are characterized by being radial.

\section{CONCLUSIONS}

In this paper, we proposed CoDistFlow to solve the nonconvex scenario-based AC OPF with intermittent renewable resources and battery storage, where current relaxation practices do not apply. CoDistFlow handles the non-convexity of the scenario-based AC OPF by iterating until convergence over an improved DistFlow approximation with correction terms for line losses and nodal voltages computed via load flows based on the battery power values obtained by the previous iteration. A fixed point of CoDistFlow is shown to satisfy the exact power flow equations and the exact operational constraints. We obtained a dispatch plan for an entire distribution network, while accounting for the grid and battery losses, and possible realizations of the stochastic resources and load. We have shown how to combine CoDistFlow with more realistic battery modeling at no extra cost in complexity. Also, we have thoroughly studied via numerical evaluations and simulations the negative effect of not considering the grid and battery losses in the dispatch plan by comparing with the DistFlow approximation in both a simple and a realistic grid. Finally, we have shown the advantages of CoDistFlow via comparisons with another iterative linearization scheme. We expect that our findings will enable the effective dispatch using storage of modern distribution grids in presence of stochastic heterogeneous resources, thus contributing to a more stable and predictable global grid. This is of significant importance as the deployment of distributed energy resources increases monotonously and may significantly impact the stability of the grid due to their stochasticity, only to make things worse when not accounting for the grid and battery losses, as shown in this paper.

\section{APPENDIX A \\ PROOF OF THEOREM 1}

Proof of Theorem 1. We define as $f^{d}(t)$ the column vector of square current magnitude values at $z_{l}$ for all lines (dimension $N)$. I, $\mathbf{0}$ are the identity and zero matrices correspondingly with dimension $N \times N$ and $0_{v}$, the zero vector with dimension $N$. Also, $r=\left[r_{1}, \ldots, r_{N}\right]^{T}, b=\left[b_{1}, \ldots, b_{N}\right]^{T}, e=[1,0, \ldots, 0]^{T}$ (dimension $N$ ), $z=\left[\left\|z_{1}\right\|^{2}, \ldots,\left\|z_{N}\right\|^{2}\right]^{T}$. Then, for scenario $d$ and time $t$ the power flow equations can be written in each corresponding case, LF, iDF, as:

a) $L F$ :

$$
\begin{array}{r}
P^{d}(t)=p^{d}(t)+p_{B}^{d}(t)+\mathbf{G} P^{d}(t)+\operatorname{diag}(r) f^{d}(t), \\
Q^{d}(t)=q^{d}(t)+q_{B}^{d}(t)+\mathbf{G} Q^{d}(t) \\
-\frac{1}{2} \operatorname{diag}(b)\left(\mathbf{I}+\mathbf{G}^{T}\right) v^{d}(t)+\operatorname{diag}(x) f^{d}(t), \\
v^{d}(t)=\mathbf{G}^{T} v^{d}(t)+v_{0} e-2 \operatorname{diag}(r) P^{d}(t) \\
-2 \operatorname{diag}(x) Q^{d}(t)-\operatorname{diag}(x) \operatorname{diag}(b) \mathbf{G}^{T} v^{d}(t) \\
+\operatorname{diag}(z) f^{d}(t), \\
f_{l}^{d}(t)=\left\|S_{l}^{d}(t)+j v_{\mathrm{up}(l)}^{d}(t) \frac{b_{l}}{2}\right\|^{2} / v_{\mathrm{up}(l)}^{d}(t) \\
\operatorname{Condition~NL1.}
\end{array}
$$

b) $i D F$ :

$$
\begin{array}{r}
P^{d}(t)=p^{d}(t)+p_{B}^{d}(t)+\mathbf{G} P^{d}(t)+\hat{P}^{d}(t), \\
Q^{d}(t)=q^{d}(t)+q_{B}^{d}(t)+\mathbf{G} Q^{d}(t) \\
-\frac{1}{2} \operatorname{diag}(b)\left(\mathbf{I}+\mathbf{G}^{T}\right) v^{d}(t)+\hat{Q}^{d}(t), \\
v^{d}(t)=\mathbf{G}^{T} v^{d}(t)+v_{0} e-2 \operatorname{diag}(r) P^{d}(t) \\
-2 \operatorname{diag}(x) Q^{d}(t)-\operatorname{diag}(x) \operatorname{diag}(b) \mathbf{G}^{T} v^{d}(t)+\hat{V}^{d}(t) .
\end{array}
$$

Then, the power flow equations within iDF (i.e., Eqs. (63)(65)) can be written more compactly as:

$(\mathbf{I}-\mathbf{R}) E^{\prime}(t, d)=\mathbf{A}\left(s(t, d)+s_{B}(t, d)\right)+C(t, d)+K$,

and the exact power flow equations (LF) are the same but subject to condition NL1, i.e.,

$$
\begin{array}{r}
(\mathbf{I}-\mathbf{R}) E(t, d)=\mathbf{A}\left(s(t, d)+s_{B}(t, d)\right)+\mathbf{D} f^{d}(t)+K, \\
f^{d}(t)=\varphi_{N}(E(t, d)),
\end{array}
$$

where $\varphi_{N}($.$) is a non-linear function defined based on NL1$ and

$$
\mathbf{R}=\left[\begin{array}{ccc}
\mathbf{G} & \mathbf{0} & \mathbf{0} \\
\mathbf{0} & \mathbf{G} & -\frac{1}{2} \operatorname{diag}(b)\left(\mathbf{I}+\mathbf{G}^{T}\right) \\
-2 \operatorname{diag}(r) & -2 \operatorname{diag}(x) & (\mathbf{I}-\operatorname{diag}(x) \operatorname{diag}(b)) \mathbf{G}^{T}
\end{array}\right],
$$

$\mathbf{A}=[\mathbf{I}, \mathbf{0} ; \mathbf{0}, \mathbf{I} ; \mathbf{0}, \mathbf{0}]$,

$K=\left[0_{v} ; 0_{v} ; v_{0} e\right]$,

$\mathbf{D}=[\operatorname{diag}(r) ; \operatorname{diag}(x) ; \operatorname{diag}(z)]$.

In [7], it is shown that Eq. (66) has a unique solution for given $C^{*}, s_{B}^{*}$, if $\left\|\mathbf{H}^{T} \mathbf{M}\right\|_{1}<1$. Note that in this case $C^{*}(t, d)=\mathbf{D} f^{d, *}(t), \forall t, d$, where $f^{d, *}(t)$ corresponds to power injections $s_{B}^{*}(t, d)$ since $\left[E(t, d), C^{*}(t, d), \tilde{V}^{*}(t, d)\right]=$ $\operatorname{LF}\left(s_{B}^{*}(t, d), s(t, d)\right)$. Thus, at the fixed point of CoDistFlow, Eqs. (66), (67) coincide and have a unique solution. This proves Theorem 2 . 


\section{APPENDIX B \\ IL-OPF ALGORITHM}

Let us define $V_{i}^{d}(t), \Theta_{i}^{d}(t), I_{l}^{d}(t)$ the voltage magnitude of node $i$, voltage angle of node $i$ and current magnitude of line $l$ respectively for scenario $d$ and time $t$, and $V^{d}(t), \Theta^{d}(t), I^{d}(t)$ the corresponding collective column vectors for all nodes or lines. Also, $J_{P Q}^{f u l l, d}(t)$ stands for the Newton-Raphson Jacobian of our system defined as in [13], $J_{P Q}^{f u l l, d}(t, j)$ stands for the $j^{t h}$ line of the Jacobian, $J_{P Q}^{d}(t)$ is the Jacobian excluding the first row and the first column that correspond to the PCC (and thus it is invertible [13]) and finally, $J_{P Q}^{-1, d}(t)$ the inverse of $J_{P Q}^{d}(t)$ with an additional row and an additional column both being the first ones and filled with zero elements. For a vector $x$, we define as $\delta x=x-x^{O}$, where $x^{O}$ refers to the operating point around which we perform the linearization. Let $y^{O}$, the collection of all $P_{1}^{d O}(t), Q_{1}^{d O}(t), V^{d O}(t), \Theta^{d O}(t), I^{d O}(t), s_{B}^{O}(t, d), \forall t, d$, and $y$ the one consisting of all $P_{1}^{d}(t), Q_{1}^{d}(t), V^{d}(t), \Theta^{d}(t), I^{d}(t), s_{B}(t, d), \forall t, d$. Then, the load flow equations are linearized as follows.

$$
\begin{array}{r}
\delta P_{1}^{d}(t)=\left.J_{P Q}^{f u l l, d}(t, 1)\right|_{y^{0}}\left[\delta V^{d}(t) ; \delta \Theta^{d}(t)\right], \\
\delta Q_{1}^{d}(t)=\left.J_{P Q}^{f u l l, d}(t, N+1)\right|_{y^{0}}\left[\delta V^{d}(t) ; \delta \Theta^{d}(t)\right], \\
{\left[\delta V^{d}(t) ; \delta \Theta^{d}(t)\right]=\left.J_{P Q}^{-1, d}(t)\right|_{y^{0}}\left[\delta p_{B}^{d}(t) ; \delta q_{B}^{d}(t)\right],} \\
\delta I^{d}(t)=\left.\left[\frac{\partial I^{d}(t)}{\partial V^{d}(t)}, \frac{\partial I^{d}(t)}{\partial \Theta^{d}(t)}\right]\right|_{y^{0}}\left[\delta V^{d}(t) ; \delta \Theta^{d}(t)\right] .
\end{array}
$$

Based on the above linearization of the load flow equations we design the L-OPF module where L stands for linearized. L$\mathrm{OPF}$ is given as $\left[S^{D P}, y\right]=\mathrm{L}-\mathrm{OPF}\left(y^{O}\right)$. Then, $\mathrm{L}-\mathrm{OPF}$ solves the following optimization problem:

$$
\begin{array}{r}
\min _{S^{D P}, y}(25), \\
\text { s.t. } \forall t, d, l, \\
(11)-(13),(17)-(22),(73)-(76), \\
\underline{v} \leq V_{i}^{d}(t) \leq \bar{v}, I_{l}^{d}(t) \leq \bar{I}_{l} .
\end{array}
$$

Then we design the iterative L-OPF algorithm denoted as iL-OPF as shown in Algorithm 2. The idea is to iteratively compute the operating point around which the linearization is performed until convergence. The operating point for the linearization of the current iteration is the optimal set $y$ obtained by the previous iteration. The initial operating point $y^{O,(0)}$ is considered known.

Note that this algorithm is valid for both radial and meshed networks as it based on the bus injection model.

\section{REFERENCES}

[1] D. Pudjianto, C. Ramsay, and G. Strbac, "Virtual power plant and system integration of distributed energy resources," IET Renewable Power Generation, vol. 1, no. 1, pp. 10-16, March 2007.

[2] E. Mashhour and S. M. Moghaddas-Tafreshi, "Bidding strategy of virtual power plant for participating in energy and spinning reserve markets Part I: problem formulation," IEEE Trans. on Power Systems, vol. 26, no. 2, pp. 949-956, May 2011.

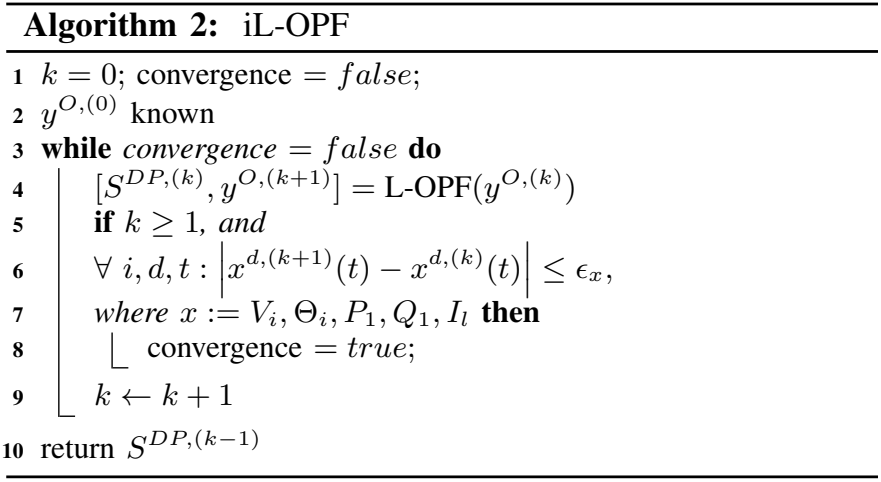

[3] F. Sossan, E. Namor, R. Cherkaoui, and M. Paolone, "Achieving the dispatchability of distribution feeders through prosumers data driven forecasting and model predictive control of electrochemical storage," IEEE Trans. on Sustainable Energy, vol. 7, no. 4, pp. 1762-1777, 2016.

[4] N. Li, L. Chen, and S. H. Low, "Exact convex relaxation of opf for radial networks using branch flow model," in IEEE Int'l Conf. on Smart Grid Com., Nov 2012, pp. 7-12.

[5] M. Farivar, C. R. Clarkey, S. H. Low, and K. M. Chandy, "Inverter VAR control for distribution systems with renewables," in IEEE Inte'l Conf. on Smart Grid Com., 2011, pp. 457-462.

[6] M. Farivar and S. H. Low, "Branch flow model: Relaxations and convexification - part i," IEEE Trans. on Power Systems, vol. 28, no. 3, pp. 2554-2564, 2013.

[7] M. Nick, R. Cherkaoui, J.-Y. L. Boudec, and M. Paolone, "An exact convex formulation of optimal power flow in radial distribution networks including transverse components," arXiv:1605.01964v2, 2016.

[8] B. Stott, J. Jardim, and O. Alsa, "DC power flow revisited," IEEE Trans. on Power Systems, vol. 24, pp. 1290-1300, 2009.

[9] D. Bienstock, M. Chertkov, and S. Harnett, "Chance-constrained optimal power flow: Risk-aware network control under uncertainty," SIAM Review, vol. 56, no. 3, pp. 461-495, 2014.

[10] M. E. Baran and F. F. Wu, "Optimal sizing of capacitors placed on a radial distribution system," IEEE Trans. on Power Delivery, vol. 4, pp. 735-743, 1989.

[11] E. Dall'Anese, K. Baker, and T. Summers, "Chance-constrained ac optimal power flow for distribution systems with renewables," IEEE Transactions on Power Systems, 2017.

[12] S. V. Dhople, S. S. Guggilam, and Y. C. Chen, "Linear approximations to ac power flow in rectangular coordinates," in 2015 53rd Annual Allerton Conference on Communication, Control, and Computing (Allerton), Sept 2015, pp. 211-217.

[13] J. Schmidli, L. Roald, S. Chatzivasileiadis, and G. Andersson, "Stochastic ac optimal power flow with approximate chance-constraints," in 2016 IEEE Power and Energy Society General Meeting (PESGM), July 2016, pp. 1-5.

[14] A. G. Bakirtzis, P. N. Biskas, C. E. Zoumas, and V. Petridis, "Optimal power flow by enhanced genetic algorithm," IEEE Trans. on Power Systems, vol. 17, pp. 229-236, 2002.

[15] E. C. Baptista, E. A. Belati, and G. R. da Costa, "Logarithmic barrieraugmented Lagrangian function to the optimal power flow problem," Int'l Journal of Electrical Power \& Energy Systems, vol. 27, pp. 528532, 2005.

[16] K. Christakou, D.-C. Tomozei, J.-Y. Le Boudec, and M. Paolone, "AC OPF in radial distribution networks - part II: an augmented Lagrangianbased OPF algorithm, distributable via primal decomposition," to appear in Electric Power Systems Research, arXiv:1503.06809v3, 2015.

[17] J. Lavaei and S. H. Low, "Zero duality gap in optimal power flow problem," IEEE Transactions on Power Systems, vol. 27, no. 1, pp. 92-107, Feb 2012.

[18] K. Christakou, D.-C. Tomozei, J.-Y. Le Boudec, and M. Paolone, "AC OPF in radial distribution networks - part I: on the limits of the Branch Flow convexification and the Alternating Direction Method of Multipliers," to appear in Electric Power Systems Research, arXiv:1503.06809v3, 2015.

[19] E. Dall'Anese, G. B. Giannakis, and B. F. Wollenberg, "Optimization of unbalanced power distribution networks via semidefinite relaxation," in 2012 North American Power Symposium (NAPS), Sept 2012, pp. 1-6. 
[20] L. Gan and S. H. Low, "Convex relaxations and linear approximation for optimal power flow in multiphase radial networks," in 2014 Power Systems Computation Conference, Aug 2014, pp. 1-9.

[21] E. Dall' Anese, H. Zhu, and G. B. Giannakis, "Distributed optimal power flow for smart microgrids," IEEE Transactions on Smart Grid, vol. 4, no. 3, pp. 1464-1475, Sept 2013.

[22] A. S. Zamzam, N. D. Sidiropoulos, and E. Dall'Anese, "Beyond relaxation and newton-raphson: Solving ac opf for multi-phase systems with renewables," IEEE Transactions on Smart Grid, 2016.

[23] Q. Peng and S. Low, "Distributed algorithm for optimal power flow on unbalanced multiphase distribution networks," arXiv, Optimization and Control (math.OC).

[24] D. Bertsimas, E. Litvinov, X. A. Sun, J. Zhao, and T. Zheng, "Adaptive robust optimization for the security constrained unit commitment problem," IEEE Trans. on Power Systems, vol. 28, no. 1, pp. 52-63, 2013.

[25] M. C. Campi and G. Calafiore, "Decision making in an uncertain environment: The scenario-based optimization approach," Working paper, Universita di Brescia, Brescia, Italy, 2004.

[26] K. Margellos, P. Goulart, and J. Lygeros, "On the road between robust optimization and the scenario approach for chance constrained optimization problems," IEEE Transactions on Automatic Control, vol. 59, no. 8, pp. 2258-2263, Aug 2014.

[27] E. Namor, F. Sossan, R. Cherkaoui, and M. Paolone, "Load leveling and dispatchability of a medium voltage active feeder through battery energy storage systems: Formulation of the control problem and experimental validation," in IEEE ISGT Europe, 2016.

[28] L. Wu, M. Shahidehpour, and Z. Li, "Comparison of scenario-based and interval optimization approaches to stochastic SCUC," IEEE Trans. on Power Systems, vol. 27, no. 2, pp. 913-921, May 2012.

[29] R. A. Jabr, S. Karaki, and J. A. Korbane, "Robust multi-period opf with storage and renewables," IEEE Trans. on Power Systems, vol. 30, no. 5, pp. 2790-2799, 2015.

[30] P. Pinson, H. Madsen, H. A. Nielsen, G. Papaefthymiou, and B. Klockl, "From probabilistic forecasts to statistical scenarios of short-term wind power production,” Wind Energy, vol. 12, no. 1, pp. 51-62, Jan. 2009.

[31] W. Zhang, F. Li, and L. M. Tolbert, "Review of reactive power planning: Objectives, constraints, and algorithms," IEEE Transactions on Power Systems, vol. 22, no. 4, pp. 2177-2186, Nov 2007.

[32] I. Koutsopoulos, T. G. Papaioannou, and V. Hatzi, "Modeling and optimization of the smart grid ecosystem," Foundations and Trends in Networking, vol. 10, no. 2-3, pp. 115-316, 2016.

[33] W. Römisch, Scenario Reduction Techniques in Stochastic Programming. Berlin, Heidelberg: Springer Berlin Heidelberg, 2009, pp. 1-14.

[34] S. Vagropoulos, E. Kardakos, C. Simoglou, A. Bakirtzis, and J. Catalão, "Ann-based scenario generation methodology for stochastic variables of electric power systems," Electric Power Systems Research, vol. 134, pp. 9-18, 2016.

[35] J.-Y. Le Boudec, Performance Evaluation of Computer and Communication Systems. EPFL Press, Lausanne, Switzerland, 2010.

[36] H. D. Chiang and M. E. Baran, "On the existence and uniqueness of load flow solution for radial distribution power networks," IEEE Transactions on Circuits and Systems, vol. 37, no. 3, pp. 410-416, Mar 1990.

[37] C. Wang, A. Bernstein, J. Y. L. Boudec, and M. Paolone, "Explicit conditions on existence and uniqueness of load-flow solutions in distribution networks," IEEE Transactions on Smart Grid, 2016.

[38] M. Dilek, F. de Leon, R. Broadwater, and S. Lee, "A robust multiphase power flow for general distribution networks," IEEE Transactions on Power Systems, vol. 25, no. 2, pp. 760-768, May 2010. 